\title{
Subterahertz and terahertz spin and lattice dynamics of the insulating ferromagnet $\mathrm{PbMnBO}_{4}$
}

\author{
M. A. Prosnikov $\odot,,^{1,2,3,{ }^{*}}$ M. E. Bal, ${ }^{1,2}$ M. I. Kolkov $\odot,{ }^{4,5}$ A. I. Pankrats $\odot,{ }^{4,5}$ R. V. Pisarev $\odot,{ }^{3}$ and P. C. M. Christianen ${ }^{1,2}$ \\ ${ }^{1}$ High Field Magnet Laboratory, EMFL, Radboud University, Toernooiveld 7, 6525 ED Nijmegen, Netherlands \\ ${ }^{2}$ Institute for Molecules and Materials, Radboud University, Heyendaalseweg 135, 6525 AJ Nijmegen, Netherlands \\ ${ }^{3}$ Ioffe Institute, 194021 St. Petersburg, Russia \\ ${ }^{4}$ Kirensky Institute of Physics, Federal Research Center KSC, Siberian Branch, Russian Academy of Sciences, 660036 Krasnoyarsk, Russia \\ ${ }^{5}$ Siberian Federal University, School of Engineering Physics and Radio Electronics, 660041 Krasnoyarsk, Russia
}

(Received 27 October 2021; revised 24 November 2021; accepted 30 November 2021; published 3 January 2022)

\begin{abstract}
Orthorhombic $\mathrm{PbMnBO}_{4}$ is a rare example of an insulating ferromagnet in which the magnetic properties, in particular the strong magnetic anisotropy, are determined by the $\mathrm{Mn}^{3+} \mathrm{Jahn}-\mathrm{Teller}$ ion. Here, we report on the detailed investigation of magnetic excitations in the subterahertz and terahertz frequency ranges, as well as lattice excitations in the terahertz range. Using polarized azimuthally resolved Raman spectroscopy, four branches of magnetic excitations were observed and assigned to one acoustic and three optical one-magnon modes. The coupling between ferromagnetic ordering at $T_{C}=31 \mathrm{~K}$ and the Jahn-Teller distortions of $\left[\mathrm{MnO}_{6}\right]$ octahedra due to the spin-phonon interaction was directly observed for some specific phonon modes involved in these distortions. A spin-wave Hamiltonian was developed that allowed us to determine a unique set of exchange and effective anisotropy parameters. The intensity ratio of the anti-Stokes to Stokes acoustic magnon peaks is found to be anomalous and directly indicates the involvement of the magneto-optical parameters in the magnetic Raman scattering process.
\end{abstract}

DOI: 10.1103/PhysRevResearch.4.013004

\section{INTRODUCTION}

A new field of magnetism, positioned as antiferromagnetic spintronics [1-5], promises to make significant progress towards higher frequencies for recording and reading information technology. However, many flourishing prospects in this field are blocked by difficulties in their practical implementation, mainly since reading information from antiferromagnets is not at all a trivial process due to the absence of a macroscopic magnetization intrinsic for ferromagnets. Additionally, the spin dynamics in ferromagnets is typically restricted to the few gigahertz to $10 \mathrm{GHz}$ frequency range, which is much lower than the subterahertz and terahertz frequency range typical for antiferromagnets.

Nonetheless, the frequency range of ferromagnets can be extended by using materials with high magnetic anisotropy, which determines the frequency of ferromagnetic resonance (FMR). Remarkably, in some ferromagnets the FMR frequency can reach the terahertz region. Notable cases are $\mathrm{CrI}_{3}$ [6,7] and $\mathrm{VI}_{3}$ [8], with FMR frequencies of 2.3 and $0.55 \mathrm{THz}$, respectively. Thus, the search for potentially useful ferromagnetic systems continues; it started with longknown metallic films $[9,10]$ and has moved to engineered

\footnotetext{
*yotungh@gmail.com

Published by the American Physical Society under the terms of the Creative Commons Attribution 4.0 International license. Further distribution of this work must maintain attribution to the author(s) and the published article's title, journal citation, and DOI.
}

heterostructures. Insulating ferromagnets are of particular interest due to the absence of the losses associated with itinerant electrons. Recently, two-dimensional limit engineering of the ferromagnetic structures was proposed in [11]. Also quasi-one-dimensional systems are appealing, such as the spin-anisotropic frustrated magnet $\mathrm{FeI}_{2}$ in which hybridized quadrupolar excitations were observed, the physics of which is governed by $S=1$ ferromagnetic chains [12]. However, even the occurrence of insulating ferromagnets is extremely rare, which undoubtedly restrains further progress on their potential applications.

One interesting example among the small group of bulk isostructural insulating magnets is $\mathrm{PbMnBO}_{4}$. It belongs to an orthorhombic $\mathrm{PbMBO}_{4}$ family, although other known members of the family with $M=\mathrm{Cr}^{3+}$ and $\mathrm{Fe}^{3+}$ are antiferromagnets. The synthesis and crystal and magnetic structures of $\mathrm{PbMnBO}_{4}$ with Curie temperature $T_{C}=31 \mathrm{~K}$ and Weiss temperature $\Theta=52.7 \mathrm{~K}$ were described, along with magnetic susceptibility, x-ray, and neutron diffraction studies, in [13]. Magnetic susceptibility, heat capacity, and FMR for $\mathrm{PbMnBO}_{4}$ were thoroughly investigated using high-quality single crystals in $[14,15]$. It was shown that the large singleion-like anisotropy of this material results in a FMR gap of $112 \mathrm{GHz}$, which is unusually high for a ferromagnet. The mixed compound $\mathrm{PbMn}_{1-x} \mathrm{Fe}_{x} \mathrm{BO}_{4}$ doped by $\mathrm{Fe}$ ions was studied in [16], which demonstrated that a small partial substitution leads to an increase of the Curie temperature, enhancement of the magnetic anisotropy, and reduction of the saturation magnetization. Nanofibers of $\mathrm{PbMnBO}_{4}$ prepared with an electrospinning method were suggested for practical application in the field of nonlinear optics as described 
in [17]. Another study observed pronounced magnetostriction and excellent magnetocaloric performance of $\mathrm{PbMnBO}_{4}$ in the vicinity of $T_{C}$ [18]. Several attempts based on the density functional theory (DFT) calculations were undertaken to predict the exchange structure $[19,20]$. Theoretical works on a nontrivial spin orientation transition and the noncollineartiy of the magnetic moments caused by the site-dependent singleion anisotropy should also be noted [21,22].

Raman scattering on the structurally identical antiferromagnet $\mathrm{PbFeBO}_{4}$ was conducted in [23], in which pronounced spin-phonon coupling was observed for some phonons; scattering on magnetic excitations, both an optical magnon and an intense two-magnon band, was observed and interpreted in terms of a linear spin-wave theory (LSWT) [24]. However, up to now, there have been no reports on lattice dynamics and spin dynamics beyond the FMR mode for $\mathrm{PbMnBO}_{4}$. Thus, the goal of the present paper is a detailed investigation of the lattice and spin dynamics as a function of temperature and static magnetic field.

This paper is organized as follows. In Sec. II the crystal and magnetic structures of $\mathrm{PbMnBO}_{4}$ are briefly reviewed. In Sec. III, the experimental details of the Raman setup for both temperature and magnetic field measurements are presented. Next, the two main sections follow for lattice dynamics (Sec. IV) and spin dynamics (Sec. V). In both sections, the polarization, temperature, and field-dependent properties of the observed excitations, phonons, and magnons are discussed in detail. Section V E is dedicated to the construction of the Heisenberg Hamiltonian with a minimal set of parameters and the derivation of the exchange and anisotropy constants based on experimentally observed magnetic excitations using linear spin-wave theory calculations. Section VI sums up and concludes the paper.

\section{CRYSTAL AND MAGNETIC STRUCTURES}

The crystal structure of $\mathrm{PbMnBO}_{4}$ is characterized by the $\mathrm{mmm}$ point group and the Pnma space group (No. 62, $Z=4$ ), and the lattice parameters determined with the use of x-ray diffraction (XRD) are $a=6.7062, b=5.9429$, and $c=9.6418 \AA$ [13]. $\mathrm{PbMnBO}_{4}$ is isostructural to $\mathrm{PbFeBO}_{4}$ and other orthoborates [25-27], which have many structural similarities, such as edge-shared oxygen octahedra forming chains running along the $b$ axis and interconnected with rigid covalent $\left[\mathrm{BO}_{3}\right]$ groups and highly distorted square pyramid $\left[\mathrm{PbO}_{4}\right]$ groups, as shown in Fig. 1. However, in contrast to both $\mathrm{PbCrBO}_{4}$ and $\mathrm{PbFeBO}_{4}$, in which $\mathrm{Cr}^{3+}$ and $\mathrm{Fe}^{3+}$ ions are in the orbitally nondegenerate state, the ground state of the $\mathrm{Mn}^{3+}$ ions is orbitally degenerate, and therefore, these ions are Jahn-Teller active, which leads to more pronounced distortion (elongation) of the $\left[\mathrm{MnO}_{6}\right]$ octahedra $[28,29]$. Nevertheless, this distortion does not lead to an overall change in crystallographic symmetry. It was previously observed in structural experiments [13] and was proposed [14] as a leading mechanism to explain the ferromagnetic ground state (contrary to the antiferromagnets $\mathrm{PbFeBO}_{4}$ and $\mathrm{PbCrBO}_{4}$ ). One can expect that a pronounced difference between the $\mathrm{Cr} / \mathrm{Fe}$ and Mn crystals should, in principle, be directly observed in Raman scattering spectra by comparing the phonons of the same symmetry.

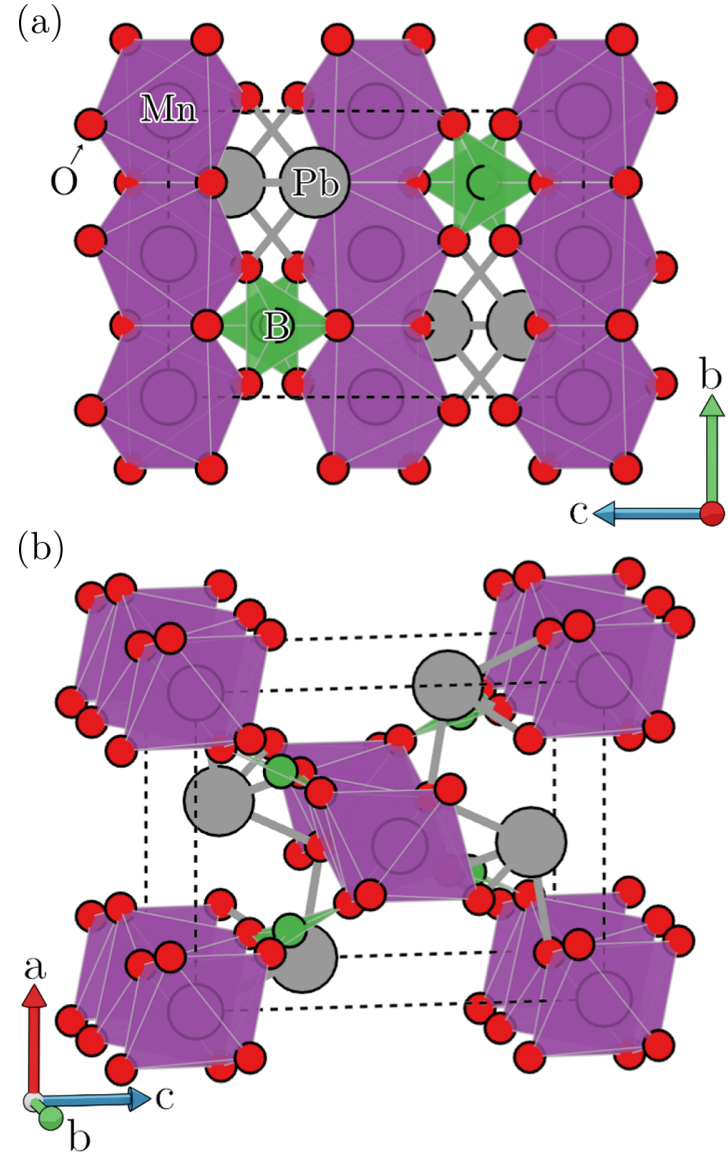

FIG. 1. Crystal structure of $\mathrm{PbMnBO}_{4}$ in polyhedral representation (a) along [100] and (b) close to [010], showing edge-shared $\left[\mathrm{MnO}_{6}\right]$ octahedra forming chains along the $b$ axis.

\section{EXPERIMENT}

High-quality single crystals used in our study come from the same batch as those used in $[14,15]$, in which a detailed description of the crystal growth process can be found. The bulk crystals are opaque, but the thin spalls are deep red colored, indicating a transparency window in the red part of the visible spectral range.

An excitation source of $660 \mathrm{~nm}$ (Thorus, LaserQuantum) was used to match the transparency window of $\mathrm{PbMnBO}_{4}$ to maximize the scattering volume, reduce laser overheating, and avoid any resonance effects in light scattering. Careful examination of the laser power effect showed that a $0.5 \mathrm{~mW}$ excitation power provides negligible overheating while still maintaining a good signal-to-noise ratio. A set of Bragg filters (Optigrate) was used in a beam splitter/rejection filters backscattering configuration. The scattered light was dispersed in a FHR1000 (HORIBA) $1 \mathrm{~m}$ spectrometer with a 1200 lines/mm grating and registered by a liquid-nitrogencooled PyLoN CCD detector (Princeton Instruments).

Temperature-dependent measurements were done using a helium flow cryostat (Oxford Instruments) with temperature stability better than $1 \mathrm{~K}$. Samples were mounted on the cold finger using silver paste. A plan achromatic long working distance objective MSPlan 50× (Olympus) was used both 
to guide the excitation light to the sample and to collect the scattered light.

Low-temperature azimuthal and magnetic field dependences were both measured in a helium bath cryostat with samples in the exchange helium gas at a base temperature of $4.2 \mathrm{~K}$. High-field experiments were performed at the High Field Magnet Laboratory (HFML, Nijmegen) in a $50 \mathrm{~mm}$ bore Florida-Bitter magnet with a maximum dc magnetic field of $30 \mathrm{~T}$. A singlet objective (with the numerical aperture of 0.68 ) with an effective focal length of $3.1 \mathrm{~mm}$ and anti-reflective coating suitable for the spectral range of interest was used for both the excitation and collection of scattered light. In all experiments, the same set of achromatic half-wave plates and Glan-Taylor polarizers (Thorlabs) were used to selectively probe specific elements of the Raman tensors. Experimental setups were controlled with the modular PYTHON suite QUDI [30].

\section{LATTICE DYNAMICS}

\section{A. Overview}

With the known structure [13], the group-theoretical analysis is applied for the determination of the number and symmetry of the possible phonon modes:

$$
\begin{aligned}
\Gamma= & 11 A_{g}+7 B_{1 g}+11 B_{2 g}+7 B_{3 g} \\
& +10 A_{u}+14 B_{1 u}+10 B_{2 u}+14 B_{3 u},
\end{aligned}
$$

where one of the whole set of $B_{1 u}, B_{2 u}$, and $B_{3 u}$ modes is acoustic phonons and all others are optical ones. Since $\mathrm{PbMnBO}_{4}$ is centrosymmetric, the mutual exclusion principle dictates that even (gerade) modes are observed only in Raman scattering and the odd (ungerade) ones are observed only in the infrared (IR) spectra. In the present Raman experiments, only even modes are probed.

Selection rules are well described with the use of the Raman tensor formalism:

$$
\begin{aligned}
A_{g} & =\left(\begin{array}{ccc}
|a| e^{i \phi_{a}} & 0 & 0 \\
0 & |b| e^{i \phi_{b}} & 0 \\
0 & 0 & |c| e^{i \phi_{c}}
\end{array}\right), \\
B_{1 g} & =\left(\begin{array}{ccc}
0 & |d| e^{i \phi_{c}} & 0 \\
|d| & 0 & 0 \\
0 & 0 & 0
\end{array}\right), \\
B_{2 g} & =\left(\begin{array}{ccc}
0 & 0 & |e| \\
0 & 0 & 0 \\
|e| & 0 & 0
\end{array}\right), \quad B_{3 g}=\left(\begin{array}{ccc}
0 & 0 & 0 \\
0 & 0 & |f| \\
0 & |f| & 0
\end{array}\right) .
\end{aligned}
$$

It should be noted that in the case of optically anisotropic noncubic crystals which possess birefringence and dichroism for the incident and scattered light and when the polarization deviates from the main crystallographic axes, the Raman tensor elements should have a complex phase factor [31-33]. For the intermediate polarization directions between the main crystallographic axes, the classical formalism (zero phase difference) predicts monotonic dependences for angles between crystallographic axes for parallel $\left(\boldsymbol{e}_{i} \| \boldsymbol{e}_{s}\right)$ configurations. However, when complex phases are taken into account, the

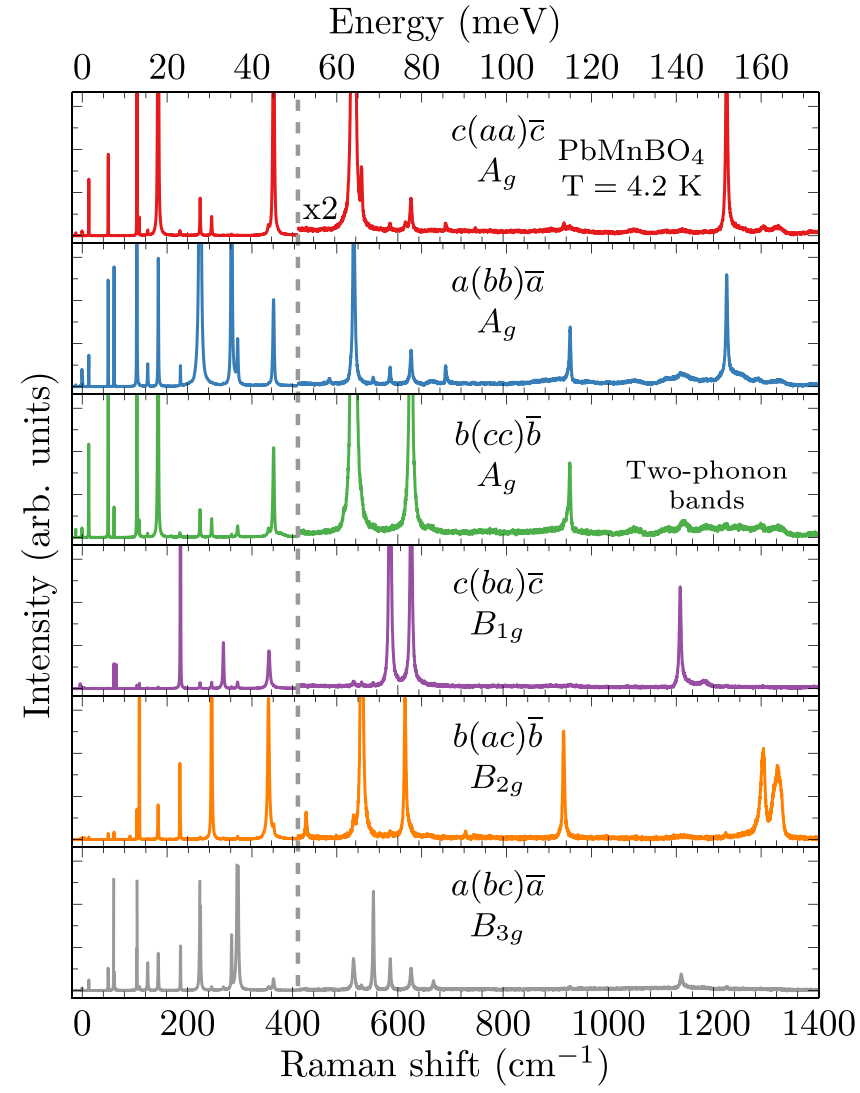

FIG. 2. Phonon spectra of $\mathrm{PbMnBO}_{4}$ for different polarizations measured at $T=4.2 \mathrm{~K}$. The intensity of the high-energy spectra above $400 \mathrm{~cm}^{-1}$ (marked by the gray vertical dashed line) is multiplied by a factor of 2 to highlight the weaker modes. Note the presence of the wide two-phonon bands at energies above $900 \mathrm{~cm}^{-1}$.

intensity behaves differently for the intermediate states of the light polarization; namely, it has additional, but still nonzero, minimum, and the intensity ratios between parallel and crossed configurations are also strongly affected. All these indicators can be directly captured in azimuthally resolved experiments.

Since the crystal structures of $\mathrm{PbMnBO}_{4}$ and $\mathrm{PbFeBO}_{4}$ are the same and the atomic masses of the $\mathrm{Mn}$ and $\mathrm{Fe}$ ions are comparable, one can expect that the phonon spectra of these two crystals should be reasonably similar. Previous studies of the lattice dynamics of $\mathrm{PbFeBO}_{4}$ using powdered samples [34] and single crystals [23] showed that the whole spectrum can be divided into three main parts: (i) the low-energy part (up to $200 \mathrm{~cm}^{-1}$ ), which should mainly correspond to phonons involving heavy $\mathrm{Pb}$ ions; (ii) the middle-energy part (200-600 $\mathrm{cm}^{-1}$ ), which is related to transition metal ions; and (iii) the high-energy part $\left(600-1300 \mathrm{~cm}^{-1}\right)$, which involves rigid covalent triangle $\left[\mathrm{BO}_{3}\right]$ groups. Mainly in the high-energy part, beginning from about $800 \mathrm{~cm}^{-1}$, a clear contribution of multiphonon scattering can be observed, consisting of broad nonsymmetric bands, additionally confirming the high structural quality of the single crystals.

As can be seen from Fig. 2, polarized Raman spectra of $\mathrm{PbMnBO}_{4}$ share most of the above-mentioned features observed in $\mathrm{PbFeBO}_{4}$. The low-energy part of the spectra 


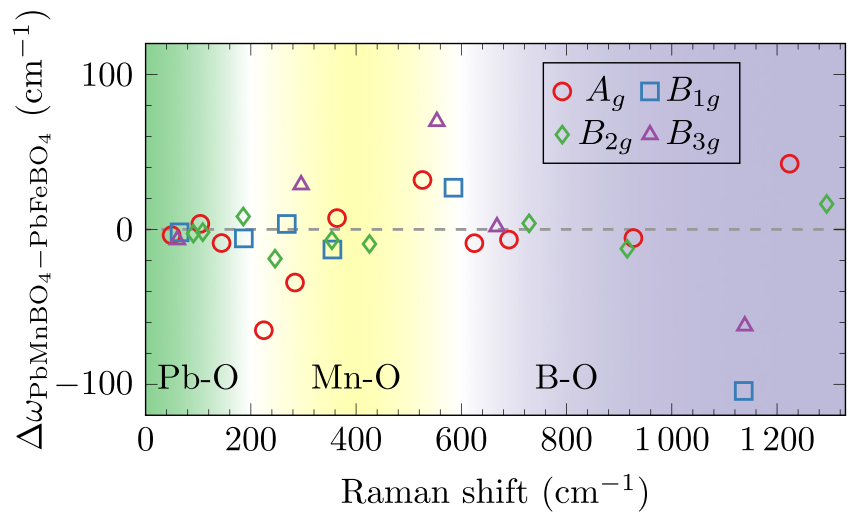

FIG. 3. Experimental results showing differences for the modes of the same symmetry between $\mathrm{PbMnBO}_{4}$ and $\mathrm{PbFeBO}_{4}$ [23]. Background colors depict low-, middle-, and high-energy regions; see text for details.

consists of a number of very sharp phonon lines, the lowest one being at $49.4 \mathrm{~cm}^{-1}$, whose linewidths are, in fact, resolution limited. The main focus in our study was placed on the low-energy part of the spectra $\left(-20-400 \mathrm{~cm}^{-1}\right)$ since we expected to observe spin dynamics exactly in this range. All temperature, polarization, and magnetic field dependences were studied in this low-energy spectral range.

The advantage of the present study of $\mathrm{PbMnBO}_{4}$ in comparison with the previous study of $\mathrm{PbFeBO}_{4}$ [23] is the ability to get direct access to all simple crystal faces, namely, (100), (010), and (001). This allowed us to achieve polarization and geometrical conditions consistent with the pure selection rules and thus undoubtedly distinguish the phonons of $B_{1 g}, B_{2 g}$, and $B_{3 g}$ symmetries. Moreover, performing azimuthal scans allowed us to further refine the symmetry assignment of overlapping modes.

All Raman lines in the spectra were fitted with the sum of Voigt peaks in order to accommodate for nonintrinsic broadening of the lines using FITYK software [35]. The extracted frequencies are listed in Table I and compared with the experimental values for $\mathrm{PbFeBO}_{4}$ [23] and DFT calculations [26]. It can be seen that the frequencies of most modes coincide well in $\mathrm{PbMnBO}_{4}$ and $\mathrm{PbFeBO}_{4}$ crystals, especially in the lowand middle- to high-energy regions; however, those phonons, the normal modes of which involve strong distortions of the $\left[\mathrm{MnO}_{6}\right]$ octahedra, significantly differ from those for similar modes in the $\mathrm{PbFeBO}_{4}$ crystal. The high-energy modes are also quite different, probably because they correspond to boron-oxygen groups connecting $\left[\mathrm{MnO}_{6}\right]$ chains and therefore are also distorted. The substantial deviation for modes corresponding to the $\left[\mathrm{MnO}_{6}\right]$ octahedra (see the yellow region in Fig. 3) is remarkable and can be used to verify the hypothesis that specific lengths and angles for the $M-\mathrm{O}-M$ bonds are responsible for entirely different ferromagnetic and antiferromagnetic ground states of $\mathrm{PbMnBO}_{4}$ and $\mathrm{PbFeBO}_{4}$, respectively, as found in the DFT calculations.

It should be noted that the detailed information on lattice dynamics is exceptionally beneficial for comparison with the results of ab initio methods since they combine multiple observables, in this case the frequencies of the 36 even phonon modes, which is way more than in the typical static
TABLE I. Frequencies $\left(\mathrm{cm}^{-1}\right)$ and symmetry of the experimentally registered phonons in $\mathrm{PbMnBO}_{4}$ with a comparison to DFT calculations [26]. Experimental data for $\mathrm{PbFeBO}_{4}$ [23] are also shown. We note that the symmetry assignment of the phonons varies in different publications although this difference can be the result of different crystallographic settings. Raman tensor elements are given for the $A_{g}(a, b, c)$ and $B_{g}(d, e, f)$ phonons according to Eq. (2). For phonons below $400 \mathrm{~cm}^{-1}$ the frequency values were directly determined by fitting the relevant polar dependences, while the frequencies of higher-energy phonons were determined only from the polarized spectra. Phase differences between diagonal elements for the $A_{g}$ modes are $\pi / 2$, while for nondiagonal ones of $B_{1 g}$ type they are $\pi / 3$ (see Fig. 6).

\begin{tabular}{|c|c|c|c|c|}
\hline $\begin{array}{l}\text { Experiment } \\
\mathrm{PbMnBO}_{4}\end{array}$ & $\begin{array}{c}\text { Mode } \\
\text { symmetry }\end{array}$ & $\begin{array}{l}\text { Tensor } \\
\text { elements }\end{array}$ & $\begin{array}{c}\text { Experiment } \\
\mathrm{PbFeBO}_{4}\end{array}$ & $\begin{array}{c}\text { DFT } \\
\mathrm{PbMnBO}_{4}\end{array}$ \\
\hline 49.4 & $A_{g}$ & $50,55,101$ & 53.1 & 52.1 \\
\hline 60.4 & $B_{3 g}$ & 140 & 67.1 & 63.6 \\
\hline 64.6 & $B_{1 g}$ & 23 & 66.5 & 65.4 \\
\hline 90.8 & $B_{2 g}$ & 11 & 93.5 & 91.4 \\
\hline 104.0 & $A_{g}$ & $130,220,210$ & 100.4 & 96.4 \\
\hline 108.6 & $B_{2 g}$ & 90 & 110.4 & 106.9 \\
\hline 144.3 & $A_{g}$ & $300,90,210$ & 153.1 & 160.0 \\
\hline \multirow[t]{2}{*}{185.6} & $B_{2 g}$ & 52 & 177.3 & 187.7 \\
\hline & $B_{3 g}$ & & 187.9 & 198.2 \\
\hline 186.7 & $B_{1 g}$ & 120 & 192.5 & 189.5 \\
\hline 224.7 & $A_{g}$ & $60,480,50$ & 289.8 & 213.9 \\
\hline 246.0 & $B_{2 g}$ & 155 & 264.9 & 226.7 \\
\hline 268.3 & $B_{1 g}$ & 64 & 264.8 & 251.4 \\
\hline 283.9 & $A_{g}$ & $20,255,30$ & 318.2 & 273.3 \\
\hline \multirow[t]{2}{*}{295.5} & $B_{3 g}$ & 160 & 266.7 & 283.2 \\
\hline & $B_{3 g}$ & & & 294.7 \\
\hline 354.0 & $B_{2 g}$ & 138 & 361.0 & 360.0 \\
\hline 355.0 & $B_{1 g}$ & 80 & 368.0 & 360.9 \\
\hline 363.8 & $A_{g}$ & $220,110,105$ & 356.5 & 363.8 \\
\hline 425.5 & $B_{2 g}$ & & 434.9 & 426.6 \\
\hline 526.2 & $A_{g}$ & & 494.3 & 524.9 \\
\hline 531.2 & $B_{2 g}$ & & & 543.1 \\
\hline 553.5 & $B_{3 g}$ & & 483.8 & 561.6 \\
\hline 585.1 & $B_{1 g}$ & & 558.2 & 599.3 \\
\hline 613.7 & $B_{2 g}$ & & & 622.0 \\
\hline 624.8 & $A_{g}$ & & 633.7 & 633.1 \\
\hline 625.4 & $B_{1 g}$ & & & 633.6 \\
\hline 667.7 & $B_{3 g}$ & & 666.0 & 685.1 \\
\hline 690.5 & $A_{g}$ & & 697.1 & 695.0 \\
\hline 728.8 & $B_{2 g}$ & & 724.9 & 732.0 \\
\hline 915.2 & $B_{2 g}$ & & 927.7 & 934.0 \\
\hline 927.1 & $A_{g}$ & & 932.7 & 947.4 \\
\hline 1136.7 & $B_{1 g}$ & & 1241.0 & 1181.3 \\
\hline 1138.7 & $B_{3 g}$ & & 1201.0 & 1183.3 \\
\hline 1224.0 & $A_{g}$ & & 1181.6 & 1280.9 \\
\hline 1294.4 & $B_{2 g}$ & & 1278.0 & 1371.3 \\
\hline
\end{tabular}

structural data for cell dimensions and ionic coordinates. Thus, this can lead to models of exceptional precision. Moreover, in most Raman experiments only the frequencies of the phonons are available, with the addition of their symmetries in polarized experiments. By performing azimuthal-resolved measurements we were able to extract Raman tensor elements for each individual phonon. Such elements can be used to 


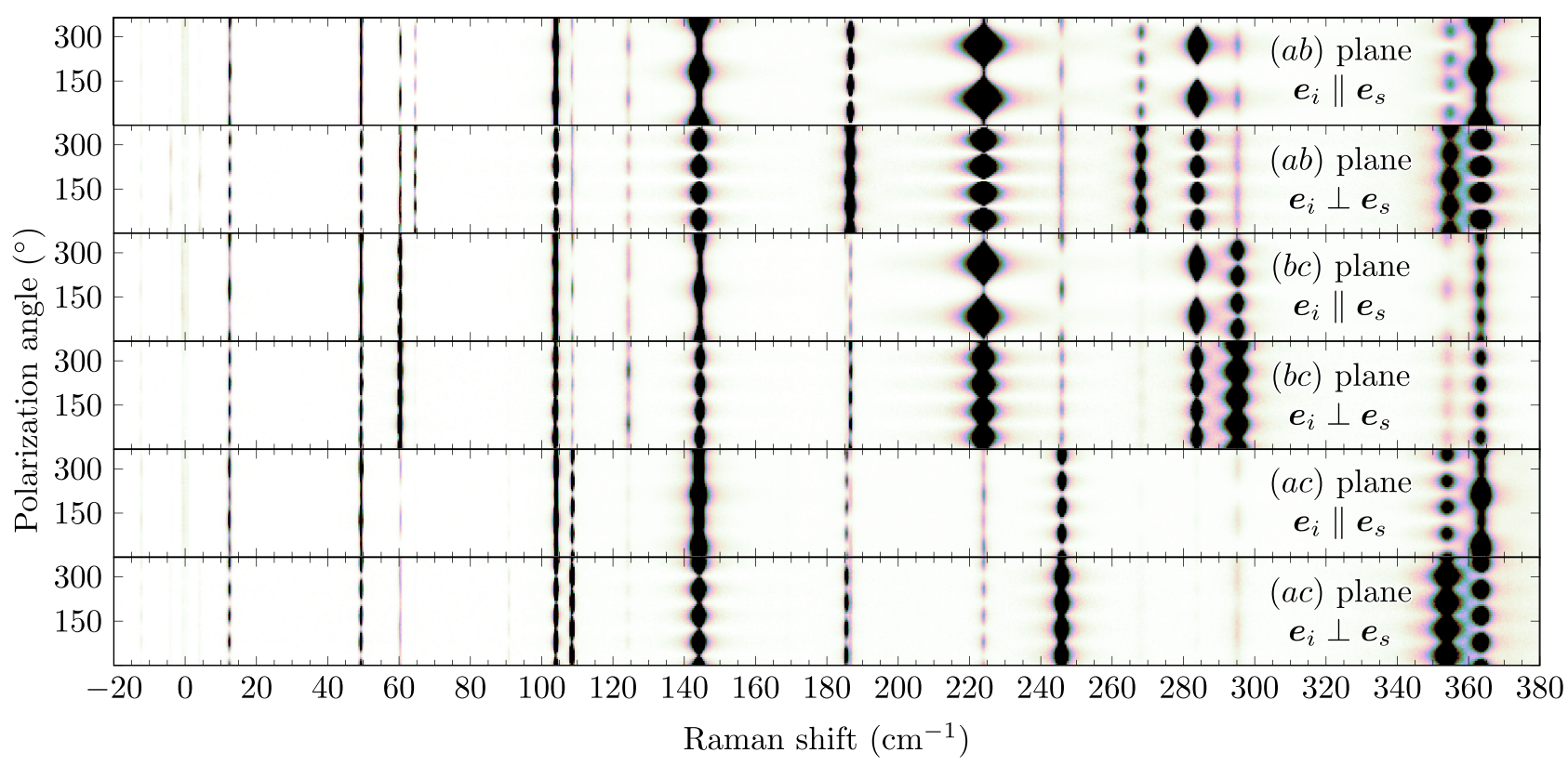

FIG. 4. Polarization-dependent intensity maps of Raman scattering spectra measured in parallel and crossed configurations in the three main, (001), (100), and (010), planes at $T=4.2 \mathrm{~K}$.

further advance DFT calculations, which in turn could shed light on the subtle aspects of the unique ferromagnetic ground state of $\mathrm{PbMnBO}_{4}$ and unveil fundamental mechanisms of the Jahn-Teller effect and magnetic interaction.

\section{B. Phonon selection rules}

The large number of the overlapping phonons with different selection rules, intensities, and half-widths impedes their correct assignment, and thus, a detailed investigation is required to determine the phonon symmetry, the contribution of complex phases, and the reliable determination of Raman tensor elements. In order to fulfill these tasks, full $360^{\circ}$ azimuthal scans were performed on the three main crystallographic faces of the $\mathrm{PbMnBO}_{4}$ single-crystal samples, and the results of this study are shown as angular-dependent spectral maps in Fig. 4. All polarization measurements reported in this section were done at $T=4.2 \mathrm{~K}$. Strong anisotropy for the in-plane angular dependences is observed as expected for the low-symmetry $\mathrm{PbMnBO}_{4}$ crystal. Minor leakage of some modes in forbidden configurations was typically less than a few percent in integrated intensity and could be explained by depolarization effects on the optical elements and in the sample itself, as well as by the use of an objective with high numerical aperture, leading to a collection of light deviating from the normal. A small deviation of the sample normal from the main crystallographic axes can also contribute to leakage and cannot be ruled out.

Despite the fact that the mode symmetry can be directly deduced from the maps, it is more informative to extract the integral angular dependence of the phonon intensity for each phonon, as shown in Figs. 5 and 6 for the $A_{g}$ and the $B_{g}$ phonons, respectively.

The angular intensity variations [31] are described according to the following equation:

$$
I=\left|\boldsymbol{e}_{i} \mathcal{R} \boldsymbol{e}_{s}\right|^{2}
$$

where $\mathcal{R}$ corresponds to a particular Raman tensor from Eq. (2) and $\boldsymbol{e}_{i}$ and $\boldsymbol{e}_{s}$ are the polarization vectors of the excitation and scattered radiation, respectively. To describe the observed azimuthal variations, these vectors are simultaneously rotated within the selected plane in parallel $\left(\boldsymbol{e}_{i} \| \boldsymbol{e}_{s}\right)$ and perpendicular (crossed; $\boldsymbol{e}_{i} \perp \boldsymbol{e}_{s}$ ) configurations. Several conclusions can be drawn immediately on the basis of only the angular-dependent polarization measurements and the data fitting using Eq. (3): (i) For most $A_{g}$ phonons, the Raman tensor elements strongly vary, and in some particular cases, e.g., for the 224.7 and $283.9 \mathrm{~cm}^{-1}$ modes, difference becomes so large that azimuthal response looks almost purely two-lobed. (ii) The intensity of the $A_{g}$ modes in the parallel configuration have well-defined minima for angles between the main crystallographic axes, which cannot be explained assuming only real Raman tensor elements. These results confirm the necessity of taking into account the complex phase formalism [31-33]. (iii) Surprisingly, the $B_{1 g}$ modes observed within the $a b$ plane should also have a complex phase between the nondiagonal elements. The effects of such complex phases are shown in the bottom right corner in Fig. 6. Notably, the nonzero phase will lead to a finite intensity in the crossed polarization geometry for angles between the crystallographic axes and to notable differences in intensity for the allowed geometries, i.e., $0^{\circ}$ and $45^{\circ}$ for crossed and parallel configurations, respectively. The limiting case of the $\pi$ phase difference which corresponds to opposite signs of the allowed nondiagonal elements manifested as finite and isotropic intensity in the crossed configuration and the absence of it in the parallel one. The observed phase differences for the $B_{1 g}$ phonons evaluated from fitting are close to $\pi / 3$, while for the diagonal elements of the $A_{g}$ modes they are equal to the expected $\pi / 2$. The Raman tensors obtained from the fitting of the experimental data can be used as the groundwork for in-depth DFT lattice dynamics calculations. One more output is the precise orientation of the $\mathrm{PbMnBO}_{4}$ single crystals 


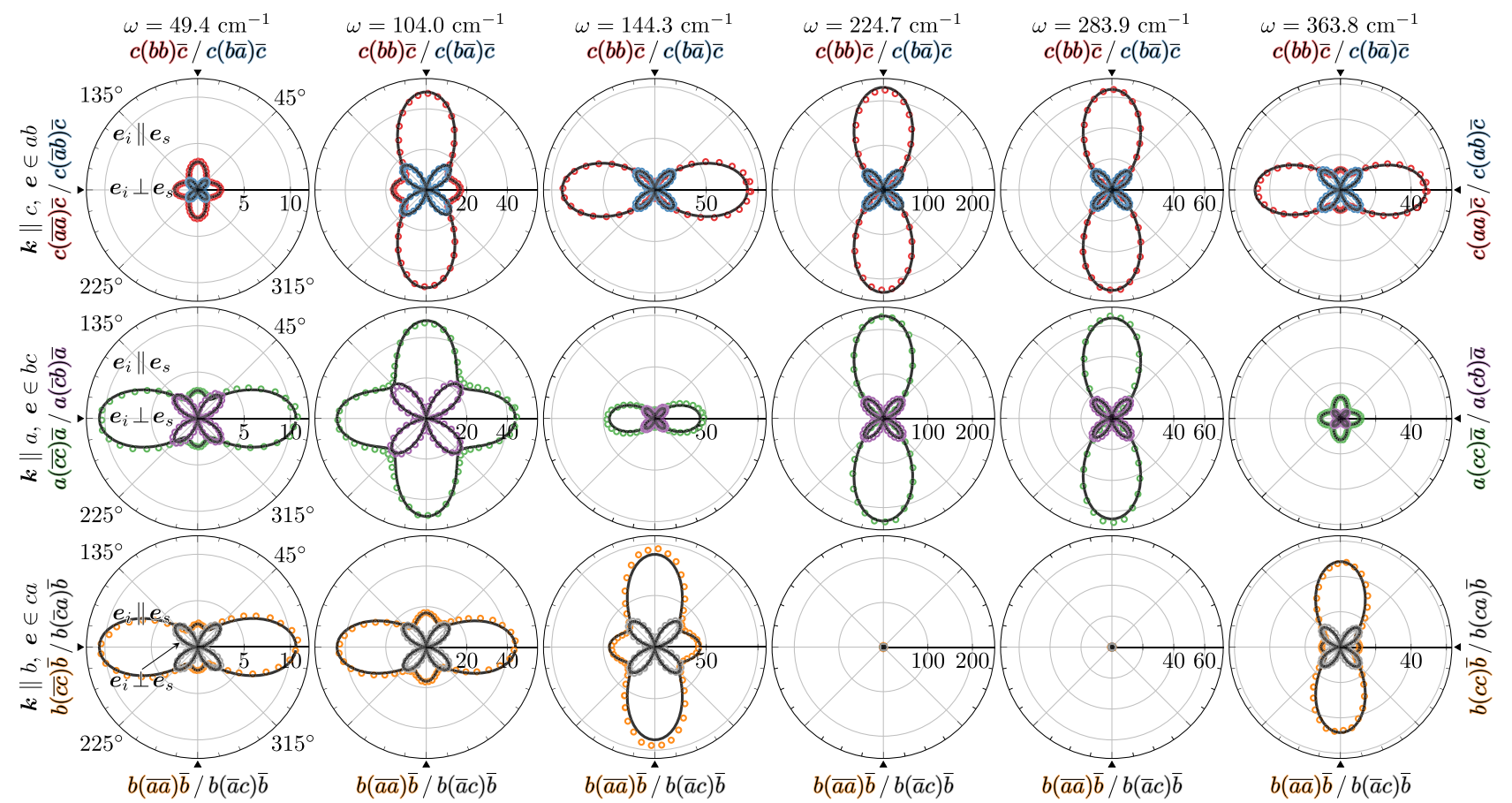

FIG. 5. Azimuthal plots of the integral phonon intensity for the $A_{g}$ modes measured at $4.2 \mathrm{~K}$. Each column corresponds to a particular phonon with the energy shown on top, and each row corresponds to a different crystal orientation. Note that the scale within each column is the same. Experimental data are shown as colored circles, and solid black lines show model calculations.

based solely on the Raman scattering study. For any arbitrary crystal orientation, the $\mathcal{R}$ tensor in Eq. (3) should be rotated according to a $R \mathcal{R} R^{-1}$ transformation, where $R$ is the rotation matrix.

\section{Temperature effects on phonons}

Temperature dependences of phonon excitations in magnetically ordered crystals allow probing the spin-phonon interaction which is typically manifested as hardening or softening of particular modes [36]. As a rule, this interaction can be conveniently described on a phenomenological level by the following equation [37]:

$$
\Delta \omega=\lambda\left\langle S_{i} S_{j}\right\rangle
$$

where the static part of the spin-spin correlation function is proportional to the square of the Brillouin function and $\lambda$ is the spin-phonon coupling coefficient. On the microscopic level,
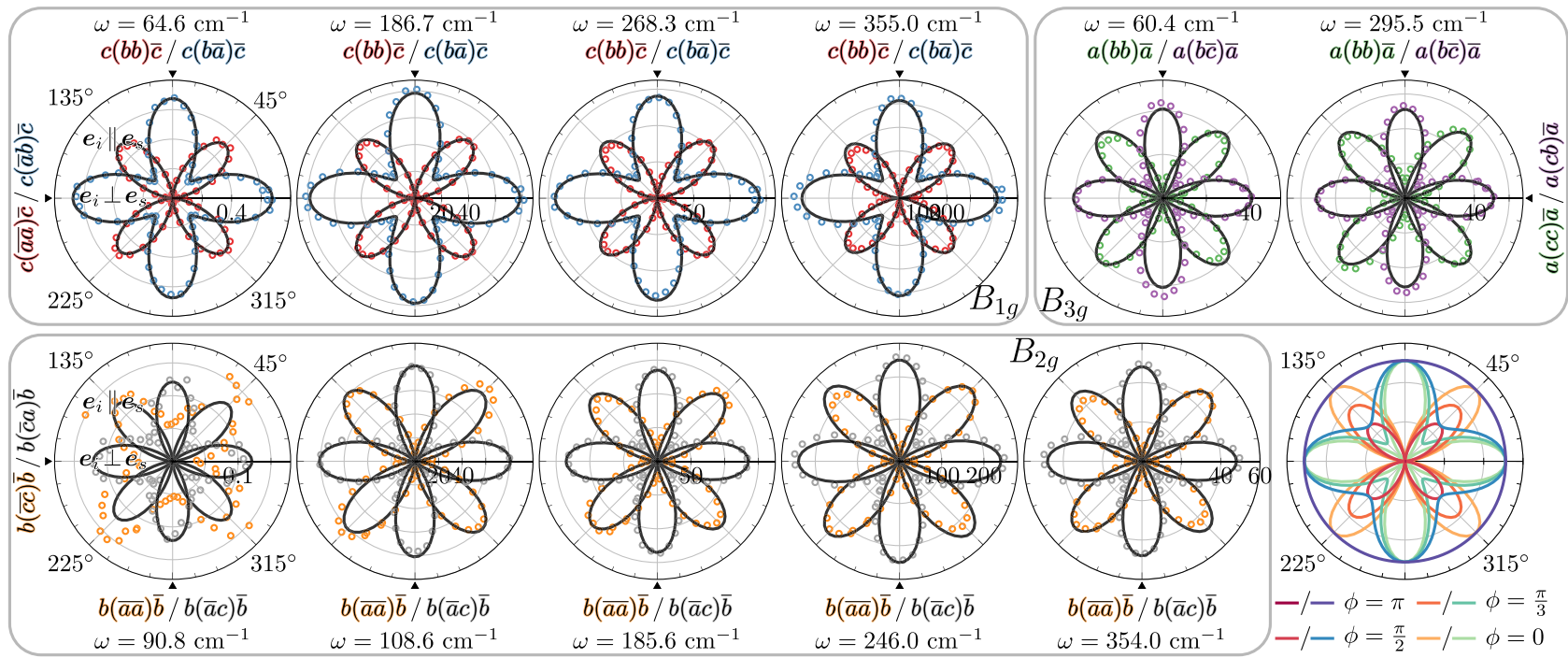

FIG. 6. Azimuthal dependences of the integral intensity of the $B_{g}$ phonons at $T=4.2 \mathrm{~K}$. In the bottom right corner, the effect of the phase difference on the nondiagonal element of the Raman tensor on the shape of the angular dependence is shown for the four different values of the apparent phase. 
the coefficient $\lambda$ should be proportional to the derivative of the exchange interaction on a normal mode coordinate because this contribution to the phonon frequency is based on the modulation of the exchange interaction by a specific phonon mode. However, since normal coordinates are inaccessible, it is impossible to estimate the spin-phonon effect on the microscopic level.

To conclusively extract the spin-phonon contribution it is necessary to disentangle it from the usual anharmonic contributions, which we will describe on a phenomenological level. This contribution affects both the phonon frequency and the half-width (inversely proportional to the lifetime) and is usually understood through three- and four-phonon relaxation processes [38]:

$$
\begin{aligned}
\omega_{i}(T)= & \omega_{i 0}-A\left(1+\frac{2}{e^{\frac{\hbar \omega_{i} 0}{2 k_{B} T}}-1}\right) \\
& -B\left(1+\frac{3}{e^{\frac{\hbar \omega_{i 0}}{3 k_{B} T}}-1}+\frac{3}{\left(e^{\frac{\hbar \omega_{i}}{3 k_{B} T}}-1\right)^{2}}\right), \\
\Gamma_{i}(T)= & \Gamma_{i 0}+C\left(1+\frac{2}{e^{\frac{\hbar \omega_{i 0}}{2 k_{B} T}}-1}\right) \\
& +D\left(1+\frac{3}{e^{\frac{\hbar \omega_{i 0}}{3 k_{B} T}}-1}+\frac{3}{\left(e^{\frac{\hbar \omega_{i} 0}{3 k_{B} T}}-1\right)^{2}}\right),
\end{aligned}
$$

where $A$ and $C$ are the constants for the three-phonon relaxation processes and $B$ and $D$ are the constants for the four-phonon relaxation processes for frequency and FWHM, respectively. The data in the paramagnetic region were fitted according to Eqs. (5) and (6). The values of $\lambda$ were extracted as the differences between an anharmonic fit and the observed frequency at $4.2 \mathrm{~K}$ [39].

Most phonons do not show any noticeable anomalies at the transition temperature [within the resolution of the setup, e.g., see Fig. 7(a)]. However, some of them in the range of 220$430 \mathrm{~cm}^{-1}$ demonstrate very pronounced frequency shifts with opposite signs. No FWHM changes could be registered, which points to a spin-phonon/exchange striction mechanism rather than to renormalization due to relaxation processes involving, for example, magnetic excitations.

Since only a small number of phonons are affected, one may assume that the origin of this effect is, indeed, the spinphonon interaction and not the change in the unit cell volume. Another argument to support this hypothesis is that most of the phonons demonstrating the spin-phonon coupling are directly related to the $\left[\mathrm{MnO}_{6}\right]$ octahedron, and this is in accordance with the DFT results obtained for $\mathrm{PbFeBO}_{4}$ [34].

Pankrats et al. [14] observed an almost complete absence of the magnetodielectric contribution in the ordered phase and concluded that $\mathrm{PbMnBO}_{4}$ has significantly weaker coupling between the lattice and magnetic subsystems in comparison with $\mathrm{PbFeBO}_{4}$ [40]. However, our observation of a pronounced spin-phonon interaction for several phonons and, even more importantly, magnetostriction effects shows the apparent interaction between these two subsystems. It is known that the magnetodielectric contribution to the dielectric susceptibility originates from the odd IR-active phonons [41]. In view of this, we can assume that mostly even Raman-active

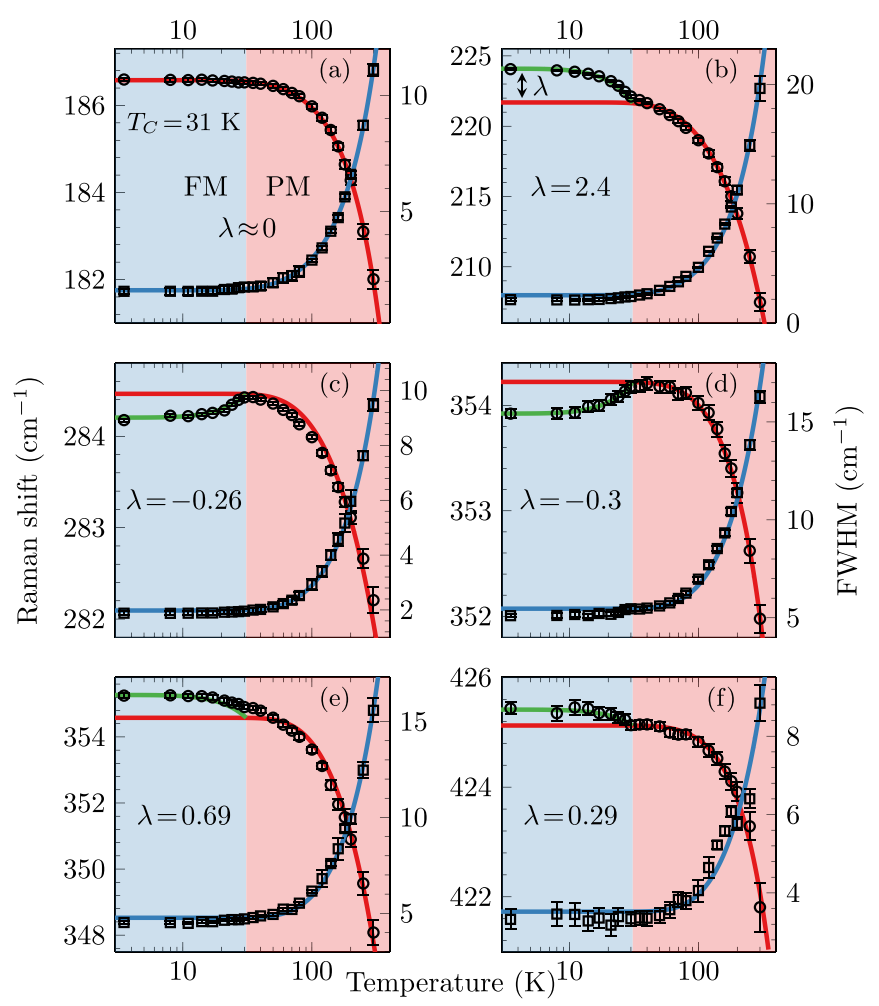

FIG. 7. Spin-phonon interaction of different phonon modes observed in the ferromagnetic phase of $\mathrm{PbMnBO}_{4}$. The logarithmic temperature scale was applied to highlight the low-temperature region.

phonons are experiencing frequency shifts due to magnetic ordering which are not manifested in the susceptibility and that careful measurements of IR-active, odd phonons would be beneficial.

Another interesting aspect is the transformation of the Raman tensor elements relevant to the $224.7 \mathrm{~cm}^{-1}$ mode as a function of temperature, in particular when crossing $T_{C}$ (see Fig. 8). Our azimuthally resolved data at $4.2 \mathrm{~K}$ and the temperature dependence of the intensity for a number of selected polarizations, for example, for the $c(a a) \bar{c}, b(a a) \bar{b}$,

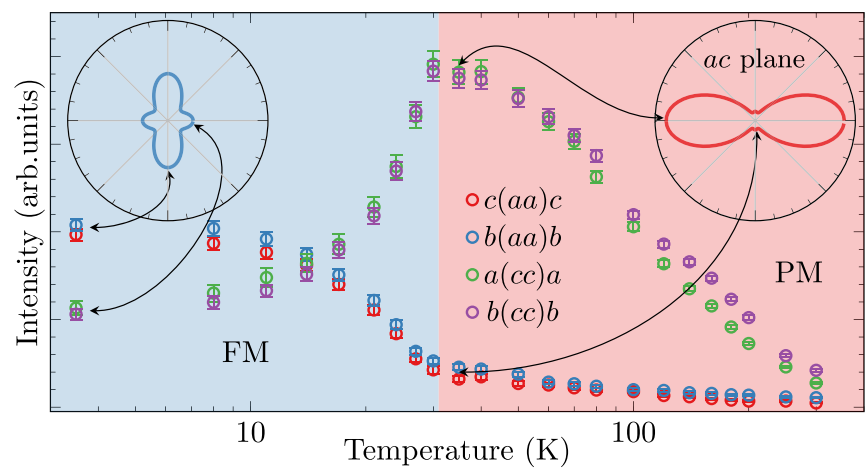

FIG. 8. Temperature (logarithmic scale) dependence of the $224.7 \mathrm{~cm}^{-1}$ phonon mode peak intensity in different polarizations. Insets demonstrate transformations of the azimuthal dependences of the Raman tensor elements deep in the ferromagnetic (blue shaded area) and paramagnetic (red shaded area) phases. 
$a(c c) \bar{a}$, and $b(a a) \bar{b}$ polarizations, allow us to restore the full and distinct transformation of the indicative surface of the Raman tensor for the $a b$ crystal plane. This effect is somewhat different from the typical manifestation of spin-phonon interaction reflected in the frequency shift. It can be associated with a contribution to the Jahn-Teller effect due to magnetic ordering because this phonon mode is directly related to the dynamics of the $\left[\mathrm{MnO}_{6}\right]$ octahedra [34]. There is no doubt that further experiments investigating odd phonons in $\mathrm{PbMnBO}_{4}$, such as infrared and terahertz spectroscopy, are necessary to shed more light on the complimentary part of the lattice dynamics and its connection to the dielectric susceptibility as well as its temperature variations. Ultrasonic measurements could be of additional interest to directly probe the uniform Jahn-Teller deformation contribution to effects observed when crossing and below $T_{C}$ [42]. Overall, the magnitude of the spin-phonon effect is weaker than in well-known $R \mathrm{MnO}_{3}$ ( $R=$ rare earth) systems [43-45]; however, the manifestation of the effect on very specific modes with opposite signs and the response to static magnetic fields will contribute to better general understanding of the spin-phonon interaction and related phenomena.

\section{Field-dependent lattice dynamics}

It was expected that that no effect of a magnetic field on the lattice subsystem would be observed due to rather weak spin-phonon coupling. However, a few modes showed subtle spectral shifts accessible due to the high resolution of the experimental setup. The observed effect is rather weak, making it necessary to mitigate the extrinsic influences such as long-term drift of the setup and slight shifts in the sample position when the field is applied. Thus, the data are presented in the following way:

$$
\Delta \omega_{i}(H)=\omega_{i}(H)-\omega_{i}(30 \mathrm{~T})-\frac{1}{N} \sum_{j \neq i} \omega_{j}(H),
$$

where $\omega_{i}(H)$ is the field dependence of the frequency of the selected mode, $\omega_{i}(30 \mathrm{~T})$ is the frequency of the same mode at $30 \mathrm{~T}$, and the last term is the mean frequency of all other phonons $N$. This normalization allowed us to evaluate and plot the effect of the magnetic field on each sufficiently narrow specific phonon mode, as shown in Fig. 9. Only the 224.7 and $283.9 \mathrm{~cm}^{-1}$ modes out of the whole set in the low-energy region $\left(<400 \mathrm{~cm}^{-1}\right)$ experience a pronounced continuous shift up to the spin-flip transition when the field is applied along the hard $c$ axis. As for the case with $H \| b$ these phonons have an intensity close to zero, as can be seen in Fig. 5, thus making it impossible to conclusively observe the shifts in Faraday geometry.

Surprisingly, these modes are the same ones that show clear spin-phonon coupling [see Figs. 7(b) and 7(c)], namely, the $224.7 \mathrm{~cm}^{-1}$ mode with $\lambda=2.4 \mathrm{~cm}^{-1}$ softens by $\approx 0.4 \mathrm{~cm}^{-1}$, while the $283.9 \mathrm{~cm}^{-1}$ mode with $\lambda=-0.26 \mathrm{~cm}^{-1}$ softens by $\approx 0.1 \mathrm{~cm}^{-1}$, suggesting a correspondence between the magnitudes but with an opposite sign. The origin of this effect can be presumably attributed to the magnetostriction when the applied magnetic field couples with the lattice, resulting in a change in the lattice parameters and, consequently, in a

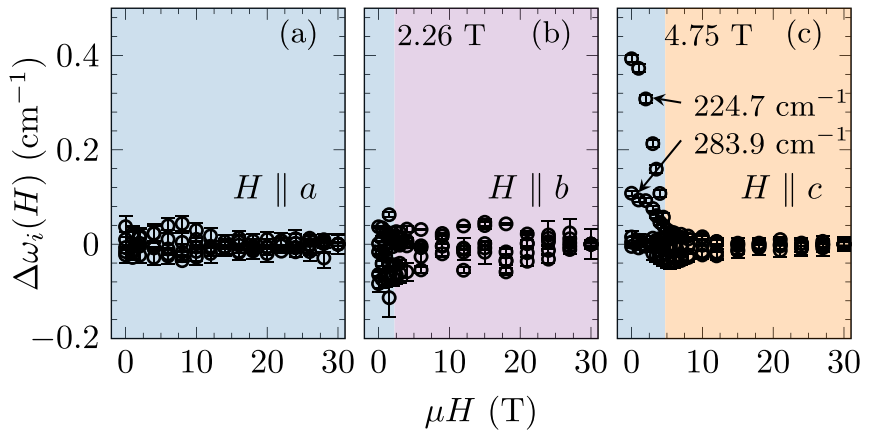

FIG. 9. Effect of an applied field on the lattice dynamics in $\mathrm{PbMnBO}_{4}$ according to Eq. (7) at $4.2 \mathrm{~K}$. Only the 224 and $284 \mathrm{~cm}^{-1}$ phonons show a pronounced effect when the field is applied along the $c$ axis. Colored regions show different magnetic phases. Values of critical fields are adopted from [14].

modification of the phonon frequency. However, this scenario seems to be unlikely because only two modes demonstrate such behavior, while the larger part of the phonon spectrum is expected to immediately reflect any change in the crystal lattice parameters with the corresponding Grüneisen parameters.

An alternative hypothesis is similar to the spin-phonon effect, which is phenomenologically described (see Sec. IV C) as an additional contribution to the phonon force constants appearing at the transition from the paramagnetic to the ordered state [e.g., ferromagnetic (FM) or antiferromagnetic (AFM)] due to the presence of magnetic order. Thus, the observed effect can be attributed to a field-dependent contribution to the spin-phonon coupling or a specific exchange-striction affecting only bonds and/or groups, most likely related to the $\left[\mathrm{MnO}_{6}\right]$ octahedra. However, the complete understanding of the observed effect requires application of additional complementary techniques such as XRD or dilatometry in order to determine the changes in the static parameters, i.e., cell constants, across $T_{C}$ and spin orientation transitions for hard and intermediate axes. Additionally, it would be nice to add thorough $a b$ initio calculations (the data presented in Sec. IV A) on top of the Grüneisen parameters for further deconvolution of the static and dynamic contributions to the phonon energy renormalization.

\section{MAGNETIC DYNAMICS}

\section{A. Overview}

At $T_{C}=31 \mathrm{~K} \mathrm{PbMnBO}_{4}$ undergoes ferromagnetic ordering [14], which necessarily results in hosting well-defined magnetic excitations. The number of branches, which are, within LSWT formalism, purely transverse, equals the number of magnetic ions in the magnetic unit cell; in our case $N=4$. Only one of the four expected modes, namely, the lowest-in-energy acoustic magnetic excitation or FMR mode, was previously observed [14] with the use of electronic spin resonance at $112 \mathrm{GHz} \approx 3.7 \mathrm{~cm}^{-1}$. In fact, a mode with such a low frequency is nearly the limit of continuous-wave Raman spectroscopy. Despite these experimental challenges we explored both the ultralow- and intermediate-frequency regions and registered the above-mentioned lowest-frequency 

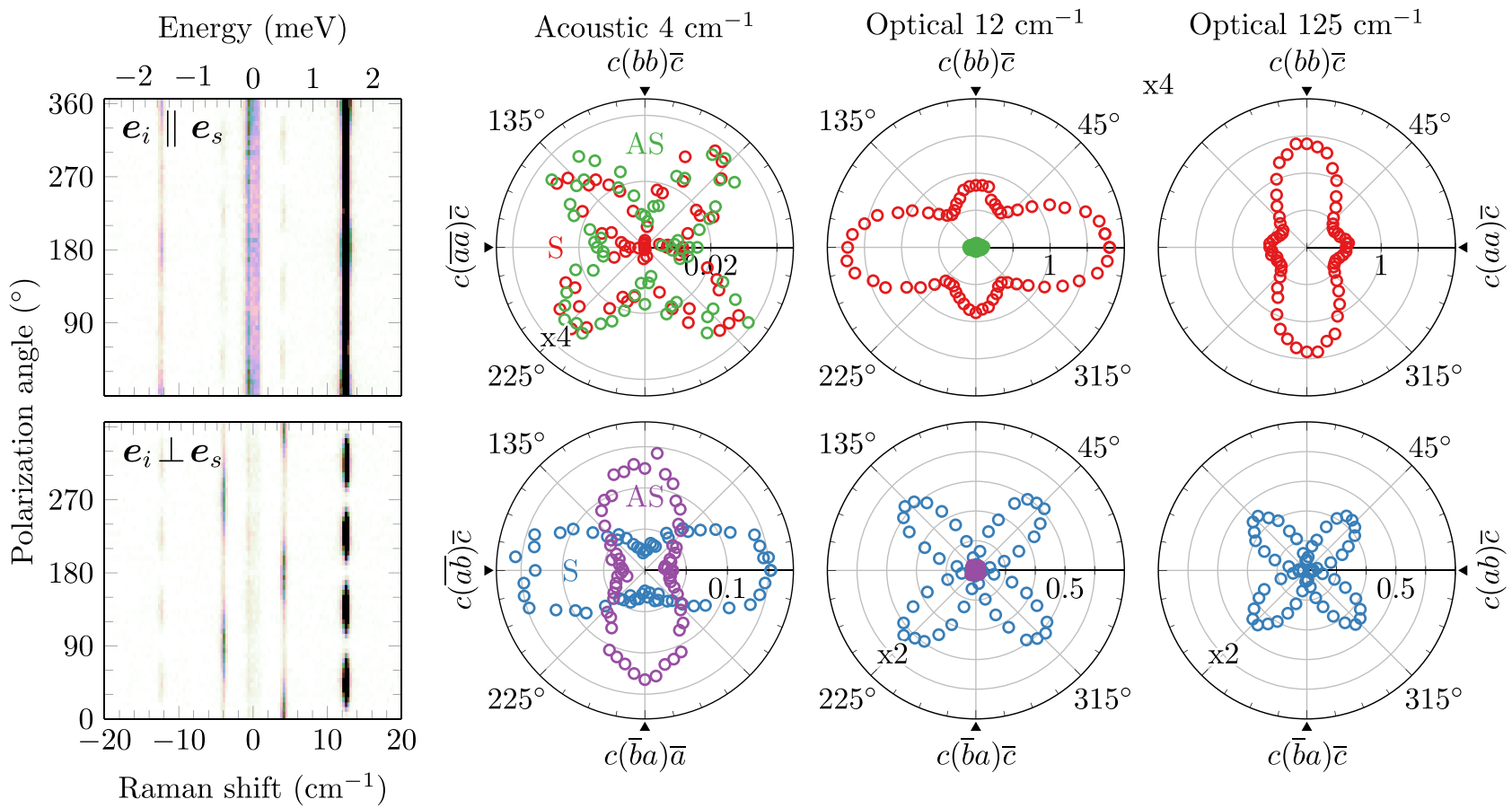

FIG. 10. Left: Azimuthal dependences of the scattered intensity in the ultralow-frequency Stokes and anti-Stokes regions for parallel (top) and crossed (bottom) configurations in the $a b$ plane. Data were not Bose corrected; see text for details. Right: Polar plots of the integral intensities for the magnon modes observed at $4.2 \mathrm{~K}$ in zero field. AS stands for modes in the anti-Stokes part, and $\mathrm{S}$ stands for the Stokes one. Note that the scale is different for some polar plots, as shown by a multiplication factor, e.g., "x2," in order to highlight particular features.

mode along with three others. To understand the origin of these modes, we used a complementary approach: First, by measuring full azimuthal dependences on the three main orthogonal faces, we were able to extract Raman tensors, revealing the mode symmetries, which are essentially different from the phonon ones. Second, we analyze the temperature dependences of observables such as frequency, full widths, etc., which also demonstrated strong deviations from the typical phonon behavior. Third, these modes manifest an expected response in an applied magnetic field. Fourth, both the frequency and field dependence of the observed excitations could be well described within a spin-wave theory formalism, as shown in Sec. VE. These observations allowed us to conclude that the four modes observed below $T_{C}$ originate from onemagnon excitations.

\section{B. Magnon polarization and selection rules}

Polarization-dependent measurement of the ultralowenergy part $\left(-20-20 \mathrm{~cm}^{-1}\right)$ at $4.2 \mathrm{~K}$ and in zero applied field showed exotic symmetries of the magnetic excitations in both the Stokes (S) and anti-Stokes (AS) regimes, as shown in Fig. 10. While the $12 \mathrm{~cm}^{-1}$ optical magnon at $4.2 \mathrm{~K}$ in zero field has a diagonal tensor of $A_{g}$ symmetry similar to phonons, the lowest- and highest-frequency magnons demonstrate very distinct and exotic responses. The intensity variation in the parallel configuration for the FMR mode looks similar to that typical for the $B_{g}$ modes with minima observed at angles corresponding to the crystal axes and maxima at intermediate angles between them (this behavior can be compared with the phonons in Fig. 6). However, in crossed configurations, instead of the expected four-lobe response, the intensity follows a twofold "dumbbell" shape displaying the intrinsic asymmetry of the Raman tensor. Moreover, the most surprising feature is the response in the crossed configuration, which looks different for the Stokes and anti-Stokes parts of the spectra. For some angles, this leads to a dramatic situation in which this mode has a substantially larger intensity in the anti-Stokes spectral part than in the Stokes one.

Similar deviations from the expected AS/S ratio were previously observed in Raman scattering experiments on the antiferromagnetic system $\mathrm{FeF}_{2}$ for which the main case of the non-Bose intensity ratio was attributed to the contribution of magneto-optical effects such as magnetic linear and circular dichroism and magnetic linear and circular birefringence $[46,47]$. We note that the observed effect was previously reported in only a few cases and at some selected angles, whereas we demonstrate its complete polarization dependence. A similar observation of AS-S asymmetry was recently found in $\mathrm{CrI}_{3}$ [7]; however, in these experiments circularly polarized light was used, which "averages out" Raman tensor elements. In our study, the use of linear polarization allowed us to directly probe these dependences.

We should note that the asymmetry effect is intrinsically different from that observed in thin ferromagnetic films $[48,49]$, in which it was associated with surface magnons, while in our study it is due to quadratic magnetooptical coupling [50].

Reconstruction of the tensors based on the observed polarization dependences of the integral intensity results in the 
following equation [50]:

$$
\frac{I_{S}}{I_{A S}}=\frac{R}{\bar{\alpha} \lambda_{e x}^{2}}\left[\left(\Psi_{\mathrm{MLB}} \mp \Psi_{\mathrm{MCB}}\right)^{2}+\left(\frac{1}{2} \Psi_{\mathrm{MLD}} \pm \frac{1}{4} \Psi_{\mathrm{MCD}}\right)^{2}\right],
$$

where $R=[n(\omega)+1] / n(\omega), n(\omega)=1 /\left(e^{\frac{\hbar \omega}{k T}}-1\right)$ is the Bose thermal population factor, $\lambda_{e x}$ is the excitation wavelength, and $\bar{\alpha}$ is the optical absorption. MLB, MCB, MLD, and MCD stand for magnetic linear birefringence, magnetic circular birefringence, magnetic linear dichroism, and magnetic circular dichroism, respectively. However, the lack of information on the magneto-optical parameters of $\mathrm{PbMnBO}_{4}$ seriously limits further progress in the interpretation of the observed effects and calls for further static magneto-optical experiments.

The selection rules of the magnetic excitations and their Raman tensors are inherently different from those for phonons and can be described by nondiagonal asymmetrical tensors with a nonzero complex phase in the following form, adapted from [51]:

$$
\begin{aligned}
M_{A_{g}} & =\left(\begin{array}{ccc}
|h| e^{i \phi_{h}} & 0 & 0 \\
0 & |m| e^{i \phi_{m}} & |p| e^{i \phi_{p}} \\
0 & |q| & |n| e^{i \phi_{n}}
\end{array}\right), \\
M_{B_{g}} & =\left(\begin{array}{ccc}
0 & |r| e^{i \phi_{r}} & |s| e^{i \phi_{s}} \\
|t| & 0 & 0 \\
|u| & 0 & 0
\end{array}\right) .
\end{aligned}
$$

The main differences from the phononic Raman tensors [Eq. (2)] are the nonsymmetric form and the simultaneous presence of both diagonal and nondiagonal elements for the $A_{g}$ mode and the occurrence of more than one nondiagonal element for the $B_{g}$ modes. It should be noted that the diagonal and nondiagonal parts of the $M_{A_{g}}$ tensors were treated separately in such a way that $I=\left|\boldsymbol{e}_{i} M_{A_{g}^{\mathrm{dg}}} \boldsymbol{e}_{S}\right|^{2}+\left|\boldsymbol{e}_{i} M_{A_{g} \text { ndg }} \boldsymbol{e}_{S}\right|^{2}$ following the approach used in [52]; such a separation of the intensity components holds for the large scattering volume, which is the case for our experiments on the bulk crystals and the use of the excitation line in the transparency window of $\mathrm{PbMnBO}_{4}$.

Tensor elements were determined by fitting the experimental data, as shown in Fig. 10, and the following parameters were obtained:

$$
\begin{aligned}
& \text { mode 4S: } r=12, t=5.3, s \approx u \approx 6.5, \\
& \text { mode 4AS: } r=5.3, t=12 \text {, } \\
& \text { mode } 12 \mathrm{~S}: h=42, m=29, n=58 \text {, } \\
& \text { mode 12AS: } h=10.5, m=7.25, n=14.5 \text {, } \\
& \text { mode 125S: } h=23, m=38, n=22, p=40, q=28 \text {, }
\end{aligned}
$$

where S and AS stand for the Stokes and anti-Stokes parts of the spectra, respectively. The phase differences between each diagonal Raman tensor element are $\pi / 2$, while those between nondiagonal elements are different and close to $120^{\circ}$ for the $M_{B_{g}}$ elements and $56^{\circ}$ between nondiagonal elements of the $M_{A_{g}}$ tensor for the $125 \mathrm{~cm}^{-1}$ optical magnon mode.

The difference in the Raman tensors could be directly linked to the symmetry of the modes itself calculated within the linear spin-wave theory, as shown in Fig. 11. The acoustic
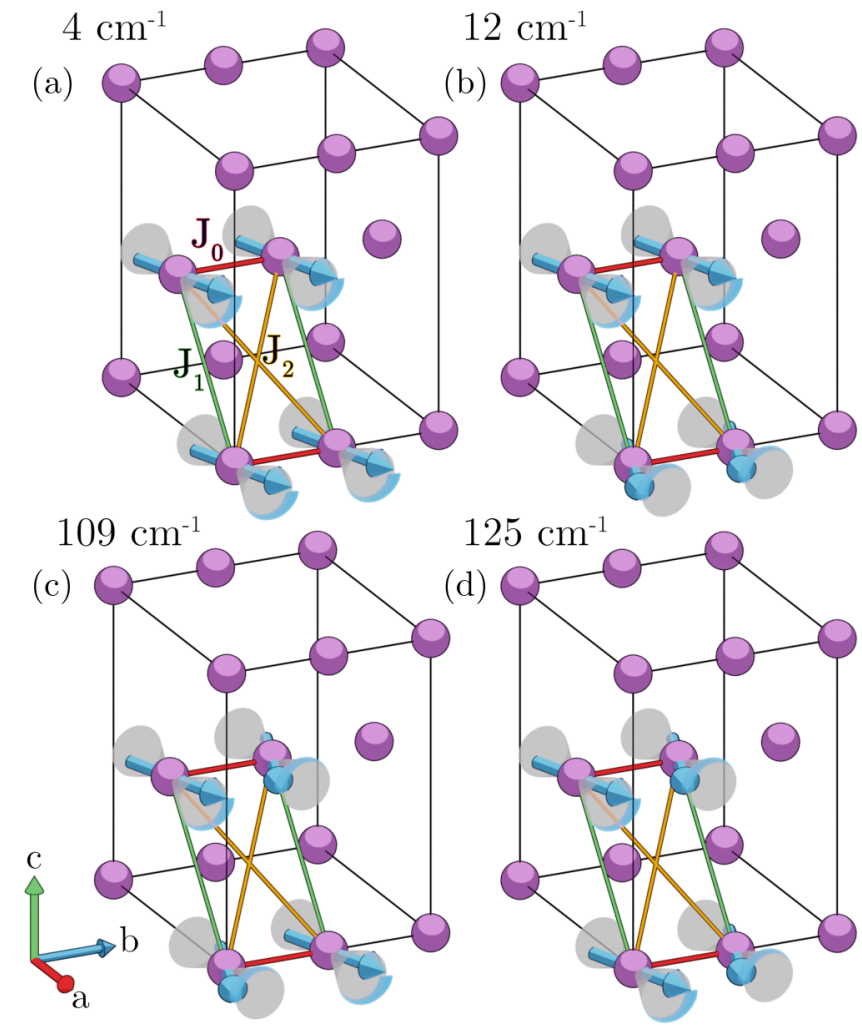

FIG. 11. Trajectories of the normal magnon modes for $\mathrm{PbMnBO}_{4}$ at the $\Gamma$ point derived from the eigenvectors of the quadratic-form Hamiltonian for the exchange parameters determined from experiments (see text for details). Only the nonequivalent part of the cell (exchange couplings and magnetic moments) is shown for clarity.

mode corresponds to the precession of the total magnetic moment around the easy $a$ axis and transforms according to the $B_{g}$ representation of the $\mathrm{mm}^{\prime} \mathrm{m}^{\prime}$ magnetic point group, while for all optical magnons there is no precession of the net moment by itself, only changes in their magnitudes.

\section{Temperature effects on spin dynamics}

The low-energy part of the spectra is dominated by the quasielastic (QE) scattering pronounced above the magnetic transition temperature, and it is strongly suppressed at low temperatures. This is followed by a complete suppression at $4.2 \mathrm{~K}$, down to an observable limit of $\approx 3 \mathrm{~cm}^{-1}$ on both the Stokes and anti-Stokes sides, as can be seen from raw data in Fig. 10. Such an observation reflects the importance of the short-range ordering effects both at and above $T_{C}$, which goes along with observations for $\mathrm{PbFeBO}_{4}$ [23] and is compatible with the hypothesis of the dimensionality crossover proposed in [24], where $\mathrm{PbFeBO}_{4}$ was considered to be a good three-dimensional antiferromagnet well below $T_{N}$. However, it shows low-dimensional features at and above $T_{N}$, namely, the presence of an anomalous peak in the magnetic susceptibility [40] and the persistence of the detectable and highly polarized QE scattering up to room temperature.

Magnon energies are proportional to the magnitudes of the magnetic moment, and their temperature changes can be typ- 


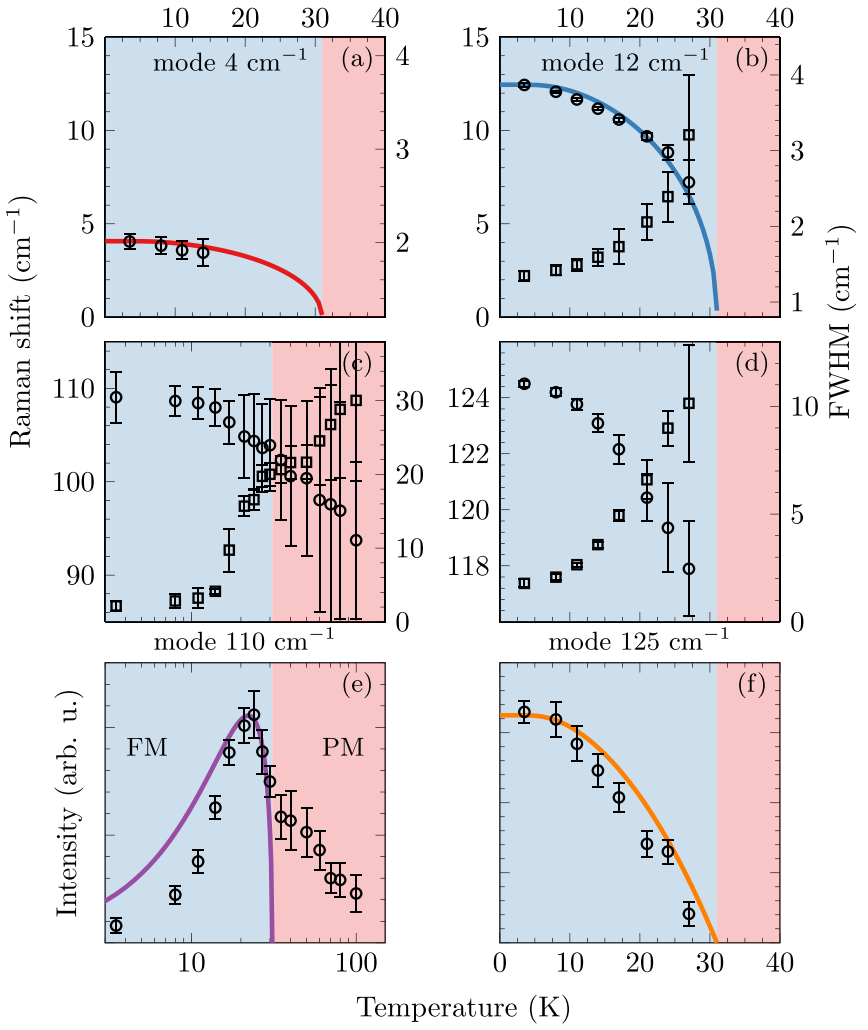

FIG. 12. Temperature dependences of (a)-(d) the energy (circles) and FWHM (squares) and (e) and (f) integral intensity of the magnetic excitations for the four observed magnetic modes. Frequencies of excitations in (a) and (b) follow a Brillouin function, while the intensity in (e) has a maximum close to a transition and is suppressed at both low and high temperatures, roughly following $\langle S S\rangle T$ dependence (purple line). Note the logarithmic temperature scale for the $110 \mathrm{~cm}^{-1}$ magnon.

ically well described by the Brillouin function. As expected, the frequency of the magnon modes is strongly temperature dependent and follows this function, as shown in Fig. 12. In contrast, all excitations have very different field and temperature dependences of the integral intensities; for example, the optical mode at $109 \mathrm{~cm}^{-1}$ is quenched at low temperatures, closely following the $\langle S S\rangle T$ function. A similar observation was made for another insulating ferromagnet, $\mathrm{CrBr}_{3}$ [53], which was later explained in [54]. This rather sharp increase in the scattering intensity is known as the critical scattering and, according to Moriya [55], is associated with fluctuations developing just below $T_{C}$. Surprisingly, all other spin-wave modes have a different intensity behavior when the temperature is varied. Namely, the intensity of the $125 \mathrm{~cm}^{-1}$ mode mainly follows a $\left\langle S^{z}\right\rangle^{2}$ function. Unfortunately, it was impossible to follow the acoustic magnon mode above $\approx 15 \mathrm{~K}$ since it softens below the rejection band of the volume Bragg grating filters $\left(\approx 3 \mathrm{~cm}^{-1}\right)$. The diversity of the temperaturedependent parameters, specifically the intensities, suggests intricate excitation and relaxation processes, potentially involving lattice dynamics, a complete understanding of which is beyond the scope of this paper.

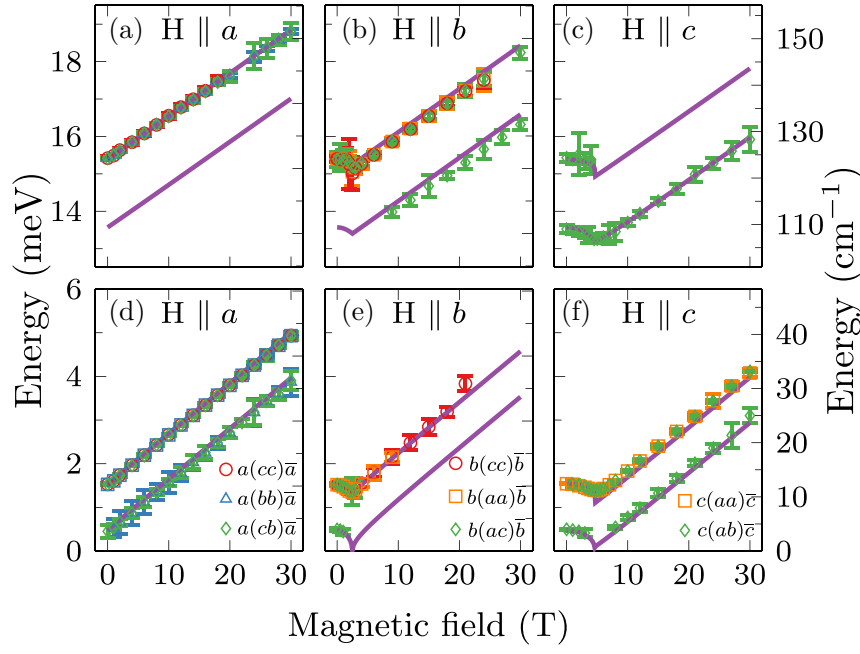

FIG. 13. Frequencies of the magnetic excitations as a function of magnetic field for the (a)-(c) high- and (d)-(f) low-energy branches. The purple line depicts the calculated field dependence based on the suggested exchange structure.

\section{Magnetic field effects on magnons}

To gain further insights into the origin of the observed modes, field-dependent measurements were performed, and the results are shown in Fig. 13. The magnetic field applied along the ferromagnetic vector ( $a$ axis) leads only to a Zeeman contribution, resulting in the nearly linear increase of the magnon frequency, acoustic and optical alike. However, a field applied perpendicular to this axis leads to a spin orientation phase transition at the field value defined by an effective anisotropy tensor. In our work, the frequencies of the magnetic excitations as a function the magnetic field were tracked, and the abrupt changes were used as markers for the determination of spin orientation transitions. In Fig. 13 such dependences revealed transitions for the intermediate (b) and hard (c) axes. These observations of spin orientation transitions and the critical field values $(2.26$ and $4.75 \mathrm{~T}$ for the $b$ and $c$ axes, respectively) are fully compatible with the FMR measurements done in [14].

\section{E. Linear spin-wave theory calculations}

Even though the energy of the acoustic magnon branch was reliably determined in [14], it was not enough to uniquely estimate the exchange constants of $\mathrm{PbMnBO}_{4}$ since the FMR frequency depends only on the anisotropy field. In contrast, Raman scattering experiments allowed us to detect all four magnetic modes corresponding to one acoustic and three optical branches. Thus, combining these observations, along with the known critical field values for spin orientation transitions, it is possible to reliably evaluate the full set of exchange and effective anisotropy constants of $\mathrm{PbMnBO}_{4}$.

Octahedral coordination of the $\mathrm{Mn}^{3+}$ ions, especially the octahedra elongation caused by the Jahn-Teller effect, could potentially lead to site-dependent anisotropy, where the local anisotropy axis follows a particular octahedra tilt. In our model we consider the site-dependent effective (single-ionlike) biaxial anisotropy constant $D$ with the local axis rotated 


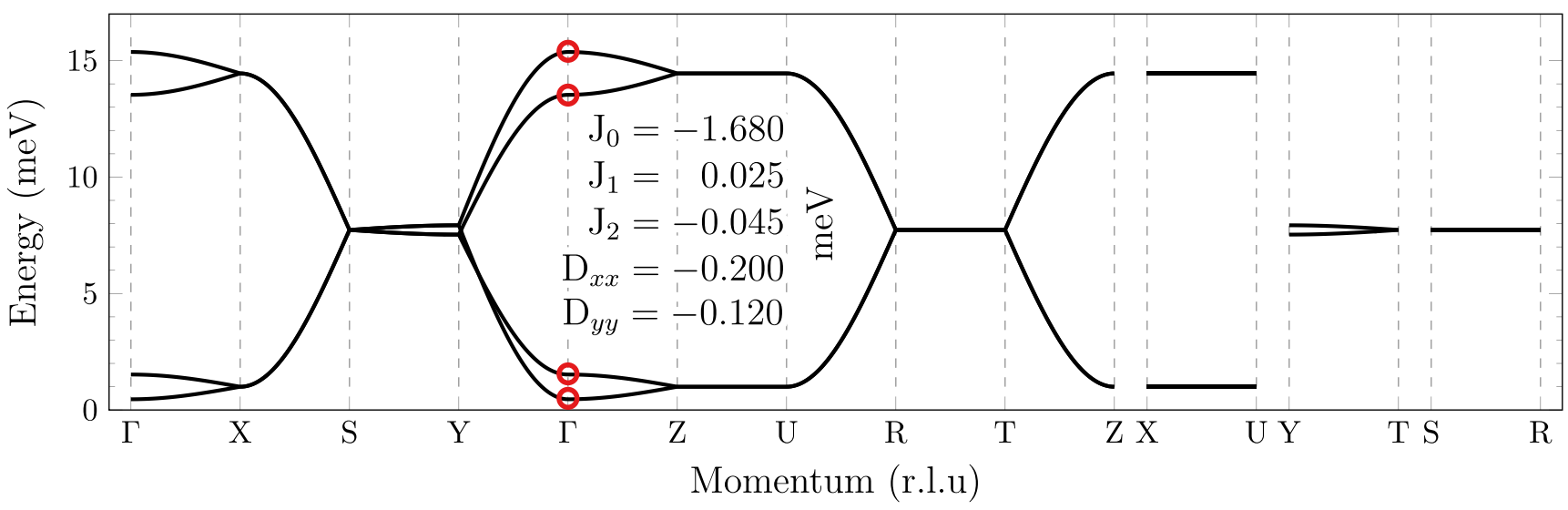

FIG. 14. Spin-wave dispersion curves between the high-symmetry points of the Brillouin zone calculated for the proposed exchange model with the constants shown in the inset (see text for details). Red circles at the $\Gamma$ point show experimentally observed values of the one-magnon modes.

by $\pm 15^{\circ}$ following rotation of the $\left[\mathrm{MnO}_{6}\right]$ octahedra within the ac plane. This step was necessary to simultaneously obtain the correct frequency of the acoustic magnon branch and reproduce the critical fields (see Fig. 13), which was impossible with considering "global" anisotropy.

It is known (and also was demonstrated for $\mathrm{PbFeBO}_{4}$ ) that the strongest exchange interaction constant $J_{0}$ corresponds to the path directed along the chains ( $b$ axis) $[19,25]$. However, even if it is unlikely, according to the phase diagram of the $J_{0}-J_{1}-J_{2}$ model [24], the ferromagnetic structure can be realized with any of these couplings being the dominating FM interaction. Thus, these will be our assumptions in determining the exchange structure.

Calculations are based on the numerical solution [56,57] of the following Heisenberg Hamiltonian:

$$
\begin{aligned}
\mathcal{H}= & \sum_{\langle i, j\rangle} J_{0} \mathbf{S}_{i} \mathbf{S}_{j}+\sum_{\langle\langle i, j\rangle\rangle} J_{1} \mathbf{S}_{i} \mathbf{S}_{j}+\sum_{\langle\langle\langle i, j\rangle\rangle\rangle} J_{2} \mathbf{S}_{i} \mathbf{S}_{j} \\
& +\sum_{i} D\left(S_{i}^{z}\right)^{2}+\mu_{B} H \sum_{i} g S_{i}^{z}
\end{aligned}
$$

where $J_{0}, J_{1}$, and $J_{2}$ are isotropic exchange constants and $D$ is the effective anisotropy tensor with $z$ along the $c$ axis.

The solution leads to four spin-wave branches nondegenerate at the $\Gamma$ point, as shown in Fig. 14. By matching the $\Gamma$-point energies with those of the experimentally registered magnons shown in Fig. 14, it is possible to derive a unique set of exchange constants, as well as the diagonal components of the anisotropy tensor $D$. The determined constants are $J_{0}=-1.68, J_{1}=0.025$, and $J_{2}=-0.045 \mathrm{meV}$ and, for the anisotropy $D$ (before the rotation), $D_{x x}=-0.20, D_{y y}=$ -0.12 , and $D_{z z}=0.0 \mathrm{meV}$, where a negative $D$ value stands for the easy-axis type of anisotropy, thus making the $a$ axis an easy axis and the $c$ axis a hard one. These values of the anisotropy tensor nicely reproduce the critical fields of the spin orientation transitions (4.6 $\mathrm{T}$ for $H \| c$ and $2.5 \mathrm{~T}$ for $H \| b$; see Fig. 13 and [14]). The full set of the exchange and anisotropy constants fully reproduces the number and the energy of the observed spin-wave branches, the small tilt of the magnetic moments even in zero field observed in [18], and a full set of magnetic field dependences.
It is curious to note that a small AFM exchange $J_{1}$ was necessary to correctly match the experimentally observed frequencies, introducing a small degree of exchange frustration. Moreover, at least three different exchange interactions are needed to match the experimental data, similar to the case of antiferromagnetic $\mathrm{PbFeBO}_{4}$ [24], making the proposed Hamiltonian the minimal one. Another interesting fact of the suggested model is that the determined $J_{0}$ is surprisingly close in magnitude to $J_{0}$ in $\mathrm{PbFeBO}_{4}$ [24] but of opposite sign, -1.68 and $1.67 \mathrm{meV}$, respectively. Thus, in these two crystals we are facing the extremely rare situation when slight structural changes (without breaking the crystallographic symmetry) induced by the Jahn-Teller distortion by $\mathrm{Mn}^{3+}$ ions lead to a sign change of the strongest exchange interaction $J_{0}$.

Symmetrically distinct normal magnon modes obtained from the eigenvectors of the quadratic form [56] of the Hamiltonian (10) are shown in Fig. 11. Slight ellipticity of the precession orbit (not shown) is expected due to the biaxial $\left(D_{x x} \neq D_{y y}\right)$ form of the anisotropy tensor. However, it less pronounced in optical modes where the isotropic exchange interactions start to contribute to the energy of the modes. The presence of the large "energy gap" at the $\Gamma$ point between the two lowest and two highest modes could be easily understood from the proposed exchange structure of $\mathrm{PbMnBO}_{4}$ shown in Fig. 11 since the lowest modes involve in-phase precession of the moments coupled by the strongest $J_{0}$ interaction, while for the highest mode the precession is in antiphase. The field dependences are sufficiently well described with $g=2.00$, suggesting the negligible role of orbital effects in the spin dynamics.

Up to now, there were no exchange constants known directly supported by experiment, e.g., inelastic neutron scattering. Nevertheless, several attempts, both experimental and theoretical, have been made to estimate the exchange structure. A $\mathrm{PbMBO}_{4}$ family of crystals was introduced in an original work by Park et al. [13] in which the magnetic susceptibility measurements were interpreted with BonnerFisher analysis [58] assuming a purely one-dimensional ferromagnetic chain, resulting in $J_{0}=-0.948 \mathrm{meV}$. Another estimate [15] was based on the experimentally determined $T_{C}$ and the paramagnetic Curie temperature $\Theta$ within 
Ginzburg-Landau theory, which resulted in $J_{0}=-1.74, J_{1}=$ $-0.19 \mathrm{meV}$ while ignoring further couplings. This estimate gives a close match in $J_{0}$ but overestimates $J_{1}$ and gives the wrong sign for it. Other attempts using $a b$ initio calculations should also be mentioned; for example, Koo and Whangbo [19] estimated the set as $J_{0}=-3.19, J_{1}=-0.043$, $J_{2}=-0.258 \mathrm{meV}$, in which the exchange was strongly overestimated. Xiang et al. obtained [20] somewhat similar values, $J_{0}=-2.321, J_{1}=-0.018, J_{2}=-0.208 \mathrm{meV}$, with all couplings being FM.

Summing up this section, we proposed a minimal Hamiltonian and a consistent exchange model with a set of exchange coupling and effective anisotropy constants of $\mathrm{PbMnBO}_{4}$ based on the frequency and field dependences of the four observed magnetic excitations and the values of critical fields of the two distinct spin orientation phase transitions. In view of the very similar crystal structures of $\mathrm{PbMnBO}_{4}$ and $\mathrm{PbFeBO}_{4}$ with almost equally strong dominating but opposite in sign $J_{0}$ exchange couplings, these systems can pave the way for manipulation of the exchange interaction $[59,60]$ or coherent control of magnetism through nonlinear phononics [61-63], e.g., through selective excitations of the specific phonon modes (see Sec. IV C) demonstrating strong coupling between lattice and magnetic subsystems.

\section{SUMMARY AND CONCLUSIONS}

In summary, a systematic inelastic Raman scattering study of the insulating ferromagnet $\mathrm{PbMnBO}_{4}$ in the wide temperature range of 4-300 $\mathrm{K}$ and in a magnetic field up to $30 \mathrm{~T}$ was presented. Most of the even Raman phonons, 34 of the symmetry-predicted 36 , were registered.

Detailed polarization-dependent measurements allowed us to undoubtedly determine their symmetry and, on the basis of the full $360^{\circ}$ azimuthal dependences, directly extract the Raman tensor elements and their complex phases. A number of modes demonstrated either softening or hardening below $T_{C}$, distinctly deviating from the usual anharmonic behavior and thus pointing to an intense spin-phonon coupling. Moreover, a $224.7 \mathrm{~cm}^{-1} A_{g}$ phonon assigned to a particular oxygen mode relevant to $\left[\mathrm{MnO}_{6}\right]$ octahedra additionally demonstrated pronounced coupling between the lattice and magnetic subsystems, manifesting in both the frequency and Raman tensor renormalization below $T_{C}$, as well as a noticeable shift in applied magnetic field. These features unravel a peculiar interaction between the Jahn-Teller elongation of the $\left[\mathrm{MnO}_{6}\right]$ octahedra and the magnetic ordering.

Four magnetic excitations were registered below $T_{C}$ on the basis of temperature and magnetic field dependences, and they all were assigned to the one-magnon scattering

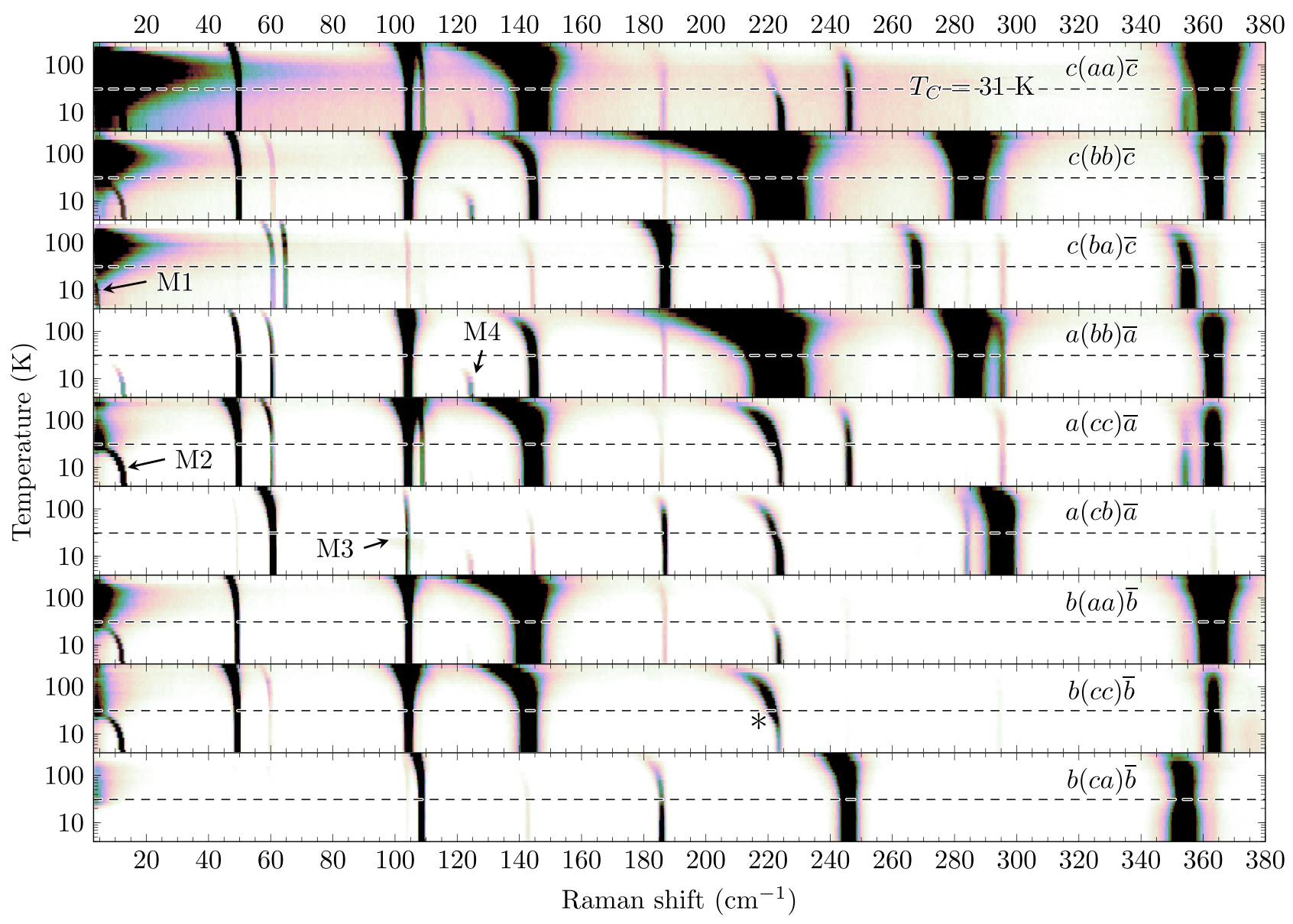

FIG. 15. Temperature-dependent Raman scattering maps for different polarizations. Observed magnons are marked M1-M4; the anomalous phonon (see Fig. 8) is marked by an asterisk. Note the logarithmic temperature scale to highlight the ferromagnetically ordered state. Horizontal dashed lines mark the PM to FM transition $\left(T_{C}\right.$, Curie) temperature. 


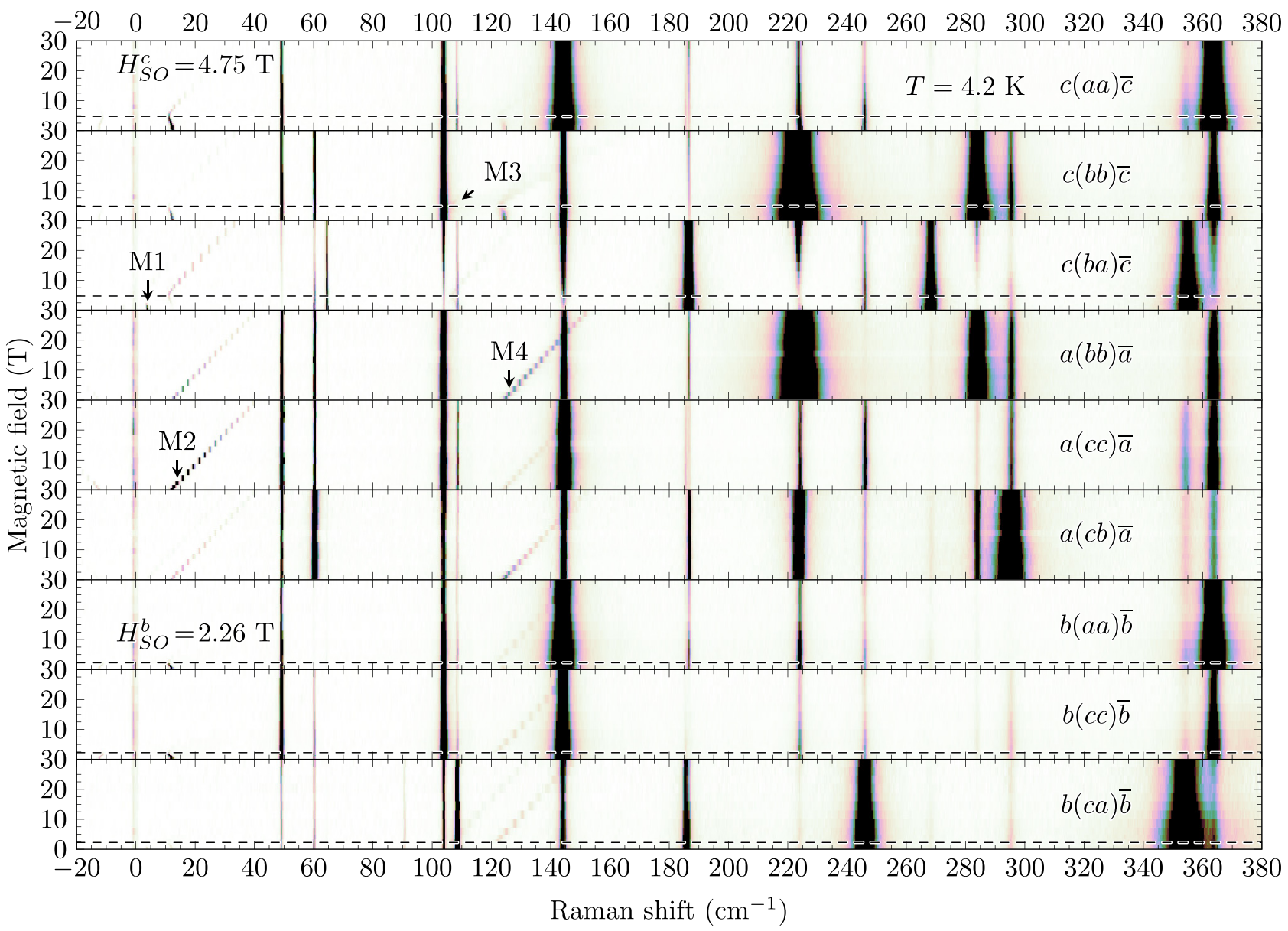

FIG. 16. Field-dependent Raman scattering maps for different polarizations. Observed magnons are marked M1-M4. Horizontal dashed lines mark spin orientation transitions. Intensity is doubled for all crossed polarizations $[c(b a) \bar{c}, a(c b) \bar{a}, b(c a) \bar{b}]$ to highlight weak magnetic excitations.

processes. Anomalous polarization-dependent Stokes to antiStokes intensity ratios of the acoustic magnon (ferromagnetic resonance) were explicitly attributed to the contribution related to magneto-optical effects. A noncontradictory set of exchange and effective anisotropy parameters was obtained based on the experimentally observed frequencies of spin excitations.

Thus, being an extremely rare example of an insulating ferromagnet, $\mathrm{PbMnBO}_{4}$ demonstrates rich spin dynamics in the subterahertz and terahertz energy range and complex features such as pronounced spin-phonon coupling, interaction of the Jahn-Teller distortion with magnetism, and a direct contribution of the magneto-optical effects to Raman scattering, making this material a rich playground for further studies of both static and dynamic properties and interaction between lattice and magnetic subsystems.

\section{ACKNOWLEDGMENTS}

M.A.P. greatly acknowledges D. A. Andronikova for aid, B. T. Crow for fruitful discussions, and P. Albers for technical assistance. Crystal growth was supported by RFBR and the Government of Krasnoyarsk Territory, Krasnoyarsk Regional Fund of Science, through Research Project No. 20-42-240006. The theoretical part of the spin dynamics calculations was supported by BASIS Foundation Grant No. 21-1-4-33-1. The support of HFML-RU/NWO-I, a member of the European Magnetic Field Laboratory (EMFL), is acknowledged.

\section{APPENDIX: RAW TEMPERATURE AND FIELD DEPENDENCE MAPS}

Two sets of the non-corrected, raw data (with only cosmic spikes removed) are shown below. Temperature dependence set shown in Fig. 15, and field dependence one in Fig. 16. Note that the different collection optics was were used along with a greatly increased distance between the sample and the spectrometer in the latter case resulting in almost complete suppression of the elastic line. 
[1] V. Baltz, A. Manchon, M. Tsoi, T. Moriyama, T. Ono, and Y. Tserkovnyak, Antiferromagnetic spintronics, Rev. Mod. Phys. 90, 015005 (2018).

[2] A. V. Chumak, V. I. Vasyuchka, A. A. Serga, and B. Hillebrands, Magnon spintronics, Nat. Phys. 11, 453 (2015).

[3] E. V. Gomonay and V. M. Loktev, Spintronics of antiferromagnetic systems, Low Temp. Phys. 40, 17 (2014).

[4] P. Němec, M. Fiebig, T. Kampfrath, and A. V. Kimel, Antiferromagnetic opto-spintronics, Nat. Phys. 14, 229 (2018).

[5] T. Jungwirth, X. Marti, P. Wadley, and J. Wunderlich, Antiferromagnetic spintronics, Nat. Nanotechnol. 11, 231 (2016).

[6] W. Jin, H. H. Kim, Z. Ye, S. Li, P. Rezaie, F. Diaz, S. Siddiq, E. Wauer, B. Yang, C. Li, S. Tian, K. Sun, H. Lei, A. W. Tsen, L. Zhao, and R. He, Raman fingerprint of two terahertz spin wave branches in a two-dimensional honeycomb Ising ferromagnet, Nat. Commun. 9, 5122 (2018).

[7] J. Cenker, B. Huang, N. Suri, P. Thijssen, A. Miller, T. Song, T. Taniguchi, K. Watanabe, M. A. McGuire, D. Xiao, and X. Xu, Direct observation of two-dimensional magnons in atomically thin $\mathrm{CrI}_{3}$, Nat. Phys. 17, 20 (2021).

[8] B. Lyu, Y. Gao, Y. Zhang, L. Wang, X. Wu, Y. Chen, J. Zhang, G. Li, Q. Huang, N. Zhang, Y. Chen, J. Mei, H. Yan, Y. Zhao, L. Huang, and M. Huang, Probing the ferromagnetism and spin wave gap in $\mathrm{VI}_{3}$ by helicity-resolved raman spectroscopy, Nano Lett. 20, 6024 (2020).

[9] V. V. Kruglyak, S. O. Demokritov, and D. Grundler, Magnonics, J. Phys. D 43, 264001 (2010).

[10] K. Neeraj, N. Awari, S. Kovalev, D. Polley, N. Zhou Hagström, S. S. P. K. Arekapudi, A. Semisalova, K. Lenz, B. Green, J.C. Deinert, I. Ilyakov, M. Chen, M. Bawatna, V. Scalera, M. d'Aquino, C. Serpico, O. Hellwig, J.-E. Wegrowe, M. Gensch, and S. Bonetti, Inertial spin dynamics in ferromagnets, Nat. Phys. 17, 245 (2021).

[11] C. Gong and X. Zhang, Two-dimensional magnetic crystals and emergent heterostructure devices, Science 363, eaav4450 (2019).

[12] X. Bai, S.-S. Zhang, Z. Dun, H. Zhang, Q. Huang, H. Zhou, M. B. Stone, A. I. Kolesnikov, F. Ye, C. D. Batista, and M. Mourigal, Hybridized quadrupolar excitations in the spinanisotropic frustrated magnet $\mathrm{FeI}_{2}$, Nat. Phys. 17, 467 (2021).

[13] H. Park, R. Lam, J. E. Greedan, and J. Barbier, Synthesis, crystal structure, crystal chemistry, and magnetic properties of $\mathrm{PbMBO}_{4}(M=\mathrm{Cr}, \mathrm{Mn}, \mathrm{Fe})$ : A new structure type exhibiting one- dimensional magnetism, Chem. Mater. 15, 1703 (2003).

[14] A. Pankrats, K. Sablina, M. Eremin, A. Balaev, M. Kolkov, V. Tugarinov, and A. Bovina, Ferromagnetism and strong magnetic anisotropy of the $\mathrm{PbMnBO}_{4}$ orthoborate single crystals, J. Magn. Magn. Mater. 414, 82 (2016).

[15] A. Pankrats, M. Kolkov, S. Martynov, S. Popkov, A. Krasikov, A. Balaev, and M. Gorev, Peculiarities of a magnetic transition in a quasi-one-dimensional ferromagnet $\mathrm{PbMnBO}_{4}$, J. Magn. Magn. Mater. 471, 416 (2019).

[16] A. Pankrats, M. Kolkov, A. Balaev, A. Shabanov, and A. Vasiliev, Forming a ferrimagnetic-like structure in the $\mathrm{PbMn}_{1-x} \mathrm{Fe}_{x} \mathrm{BO}_{4}(\mathrm{x} \approx 0.1)$ single crystal upon partial substitution, J. Magn. Magn. Mater. 497, 165997 (2020).

[17] A. Rüşen, M. A. Topçu, A. Sarilmaz, and F. Özel, Fabrication and characterization of electrospun single-crystal lead manganese borate nanofibers, Mater. Res. Bull. 99, 249 (2018).
[18] J. Head, P. Manuel, F. Orlandi, M. Jeong, M. R. Lees, R. $\mathrm{Li}$, and C. Greaves, Structural, magnetic, magnetocaloric, and magnetostrictive properties of $\mathrm{Pb}_{1-x} \mathrm{Sr}_{x} \mathrm{MnBO}_{4}(\mathrm{x}=0,0.5$, and 1.0), Chem. Mater. 32, 10184 (2020).

[19] H.-J. Koo and M.-H. Whangbo, Density functional investigation of the magnetic properties of $\mathrm{PbMBO}_{4}(M=\mathrm{Cr}, \mathrm{Mn}, \mathrm{Fe})$, Solid State Commun. 149, 602 (2009).

[20] H. Xiang, Y. Tang, S. Zhang, and Z. He, Intra-chain superexchange couplings in quasi-1D $3 d$ transition-metal magnetic compounds, J. Phys.: Condens. Matter 28, 276003 (2016).

[21] S. N. Martynov, Single-ion weak antiferromagnetism and spinflop transition in a two-sublattice ferromagnet, Phys. Solid State 62, 1165 (2020).

[22] S. N. Martynov, Ground state of the two-sublattice anisotropic ferromagnet in magnetic field, Phys. Solid State 63, 1090 (2021).

[23] M. A. Prosnikov, A. N. Smirnov, V. Y. Davydov, K. A. Sablina, and R. V. Pisarev, Lattice and magnetic dynamics of a quasione-dimensional chain antiferromagnet $\mathrm{PbFeBO}_{4}$, J. Phys.: Condens. Matter 29, 025808 (2016).

[24] M. A. Prosnikov, One- and two-magnon excitations in the antiferromagnet $\mathrm{PbFeBO}_{4}$, Phys. Rev. B 103, 094443 (2021).

[25] M. Curti, C. B. Mendive, T. Bredow, M. M. Murshed, and T. M. Gesing, Structural, vibrational and electronic properties of $\mathrm{SnMBO}_{4}(M=\mathrm{Al}, \mathrm{Ga})$ : A predictive hybrid DFT study, J. Phys.: Condens. Matter 31, 345701 (2019).

[26] T. M. Gesing, C. B. Mendive, M. Curti, D. Hansmann, G. Nénert, P. E. Kalita, K. E. Lipinska, A. Huq, A. L. Cornelius, and M. M. Murshed, Structural properties of mullite-type $\mathrm{Pb}\left(\mathrm{Al}_{1-x} \mathrm{Mn}_{x}\right) \mathrm{BO}_{4}$, Z. Kristallogr. - Cryst. Mater. 228, 532 (2013).

[27] H. Park and J. Barbier, $\mathrm{PbGaBO}_{4}$, an orthoborate with a new structure-type, Acta Crystallogr., Sect. E 57, i82 (2001).

[28] K. I. Kugel' and D. I. Khomskii, The Jahn-Teller effect and magnetism: Transition metal compounds, Sov. Phys. Usp. 25, 231 (1982).

[29] M. D. Sturge, The Jahn-Teller effect in solids, Solid State Phys. 20, 91 (1968).

[30] J. M. Binder, A. Stark, N. Tomek, J. Scheuer, F. Frank, K. D. Jahnke, C. Müller, S. Schmitt, M. H. Metsch, T. Unden, T. Gehring, A. Huck, U. L. Andersen, L. J. Rogers, and F. Jelezkoac, Qudi: A modular python suite for experiment control and data processing, Software X 6, 85 (2017).

[31] C. Kranert, C. Sturm, R. Schmidt-Grund, and M. Grundmann, Raman Tensor Formalism for Optically Anisotropic Crystals, Phys. Rev. Lett. 116, 127401 (2016).

[32] M. Grundmann, C. Sturm, C. Kranert, S. Richter, R. SchmidtGrund, C. Deparis, and J. Zúñiga-Pérez, Optically anisotropic media: New approaches to the dielectric function, singular axes, microcavity modes and Raman scattering intensities, Phys. Status Solidi RRL 11, 1600295 (2017).

[33] W. Zheng, J. Yan, F. Li, and F. Huang, Elucidation of "phase difference" in Raman tensor formalism, Photon. Res. 6, 709 (2018)

[34] M. M. Murshed, C. B. Mendive, M. Curti, G. Nénert, P. E. Kalita, K. Lipinska, A. L. Cornelius, A. Huq, and T. M. Gesing, Anisotropic lattice thermal expansion of $\mathrm{PbFeBO}_{4}$ : A study by $\mathrm{X}$-ray and neutron diffraction, Raman spectroscopy and DFT calculations, Mater. Res. Bull. 59, 170 (2014). 
[35] M. Wojdyr, Fityk: A general-purpose peak fitting program, J. Appl. Crystallogr. 43, 1126 (2010).

[36] M. G. Cottam and D. J. Lockwood, Spin-phonon interaction in transition-metal difluoride antiferromagnets: Theory and experiment, Low Temp. Phys. 45, 78 (2019).

[37] D. J. Lockwood and M. G. Cottam, The spin-phonon interaction in $\mathrm{FeF}_{2}$ and $\mathrm{MnF}_{2}$ studied by Raman spectroscopy, J. Appl. Phys. 64, 5876 (1988).

[38] M. Balkanski, R. F. Wallis, and E. Haro, Anharmonic effects in light scattering due to optical phonons in silicon, Phys. Rev. B 28, 1928 (1983).

[39] The anharmonic constants obtained through the fitting procedure are $\quad \omega_{0}=187.1, A=0.45, B=0.09, \Gamma_{0}=$ $0.35, C=1.09, D=0.17 ; \quad \omega_{0}=226.6, A=4.94, B=$ $0.02, \Gamma_{0}=0.01, C=1.78, D=0.55 ; \quad \omega_{0}=285.7, A=$ $1.25, B=0.00, \Gamma_{0}=0.01, C=1.68, D=0.30 ; \quad \omega_{0}=$ 355.0, $A=0.65, B=0.14, \Gamma_{0}=0.01, C=5.05, D=0.42$; $\omega_{0}=359.2, A=4.64, B=0.00, \Gamma_{0}=0.01, C=4.27, D=$ 0.49; $\quad \omega_{0}=426.2, A=0.72, B=0.36, \Gamma_{0}=0.01, C=$ $3.29, D=0.23$ for curves (a)-(f) in Fig. 7, respectively.

[40] A. Pankrats, K. Sablina, D. Velikanov, A. Vorotynov, O. Bayukov, A. Eremin, M. Molokeev, S. Popkov, and A. Krasikov, Magnetic and dielectric properties of the $\mathrm{PbFeBO}_{4}$ single crystal, J. Magn. Magn. Mater. 353, 23 (2014).

[41] R. M. Dubrovin, N. V. Siverin, P. P. Syrnikov, N. N. Novikova, K. N. Boldyrev, and R. V. Pisarev, Lattice dynamics and microscopic mechanisms of the spontaneous magnetodielectric effect in the antiferromagnetic fluoroperovskites $\mathrm{KCoF}_{3}$ and $\mathrm{RbCoF}_{3}$, Phys. Rev. B 100, 024429 (2019).

[42] C. Zhu and R. Zheng, Ultrasonic evidence for magnetoelastic coupling in $\mathrm{La}_{0.60} \mathrm{Y}_{0.07} \mathrm{Ca}_{0.33} \mathrm{MnO}_{3}$ perovskites, Phys. Rev. B 59, 11169 (1999).

[43] J. Laverdière, S. Jandl, A. A. Mukhin, V. Y. Ivanov, V. G. Ivanov, and M. N. Iliev, Spin-phonon coupling in orthorhombic $R \mathrm{MnO}_{3}(R=\mathrm{Pr}, \mathrm{Nd}, \mathrm{Sm}, \mathrm{Eu}, \mathrm{Gd}, \mathrm{Tb}, \mathrm{Dy}, \mathrm{Ho}, \mathrm{Y}):$ A Raman study, Phys. Rev. B 73, 214301 (2006).

[44] M. Mochizuki, N. Furukawa, and N. Nagaosa, Theory of spin-phonon coupling in multiferroic manganese perovskites $\mathrm{RMnO}_{3}$, Phys. Rev. B 84, 144409 (2011).

[45] S. Petit, F. Moussa, M. Hennion, S. Pailhès, L. Pinsard-Gaudart, and A. Ivanov, Spin Phonon Coupling in Hexagonal Multiferroic $\mathrm{YMnO}_{3}$, Phys. Rev. Lett. 99, 266604 (2007).

[46] P. A. Bates, M. G. Cottam, and S. R. P. Smith, "Anomalous" anti-Stokes:Stokes Raman scattering intensity ration in antiferromagnetic $\mathrm{FeF}_{2}$, J. Magn. Magn. Mater. 15-18, 771 (1980).

[47] P. A. Bates, M. G. Cottam, and S. R. P. Smith, "Anomalous" behaviour of the anti-Stokes-Stokes intensity ratio for one- magnon Raman scattering in $\mathrm{FeF}_{2}$, Solid State Commun. 33, 129 (1980).

[48] R. E. Camley and M. Grimsditch, Brillouin scattering from magnons in ferromagnetic thin films of polycrystalline iron, Phys. Rev. B 22, 5420 (1980).

[49] R. E. Camley, P. Grünberg, and C. M. Mayr, Stokes-anti-Stokes asymmetry in Brillouin scattering from magnons in thin ferromagnetic films, Phys. Rev. B 26, 2609 (1982).

[50] W. Wettling, M. G. Cottam, and J. R. Sandercock, The relation between one-magnon light scattering and the complex magnetooptic effects in YIG, J. Phys. C 8, 211 (1975).

[51] A. P. Cracknell, Scattering matrices for the Raman effect in magnetic crystals, J. Phys. C 2, 500 (1969).

[52] C. Kranert, C. Sturm, R. Schmidt-Grund, and M. Grundmann, Raman tensor elements of $\beta-\mathrm{Ga}_{2} \mathrm{O}_{3}$, Sci. Rep. 6, 35964 (2016).

[53] J. R. Sandercock, A light scattering study of the ferromagnet $\mathrm{CrBr}_{3}$, Solid State Commun. 15, 1715 (1974).

[54] R. Loudon, Theory of the temperature dependence of first-order light scattering by ordered spin systems, J. Phys. C 3, 872 (1970).

[55] T. Moriya, Theory of absorption and scattering of light by magnetic crystals, J. Appl. Phys. 39, 1042 (1968).

[56] S. Toth and B. Lake, Linear spin wave theory for single-Q incommensurate magnetic structures, J. Phys.: Condens. Matter 27, 166002 (2015)

[57] R. Fishman, J. Fernandez-Baca, and T. Rõõm, Spin-Wave Theory and Its Applications to Neutron Scattering and $\mathrm{THz}$ Spectroscopy (Morgan and Claypool, San Rafael, CA, 2018).

[58] J. C. Bonner and M. E. Fisher, Linear magnetic chains with anisotropic coupling, Phys. Rev. 135, A640 (1964).

[59] J. H. Mentink, Manipulating magnetism by ultrafast control of the exchange interaction, J. Phys.: Condens. Matter 29, 453001 (2017).

[60] R. V. Mikhaylovskiy, E. Hendry, A. Secchi, J. H. Mentink, M. Eckstein, A. Wu, R. V. Pisarev, V. V. Kruglyak, M. I. Katsnelson, T. Rasing, and A. V. Kimel, Ultrafast optical modification of exchange interactions in iron oxides, Nat. Commun. 6, 8190 (2015).

[61] M. Först, C. Manzoni, S. Kaiser, Y. Tomioka, Y. Tokura, R. Merlin, and A. Cavalleri, Nonlinear phononics as an ultrafast route to lattice control, Nat. Phys. 7, 854 (2011).

[62] M. Först, R. Mankowsky, and A. Cavalleri, Mode-selective control of the crystal lattice, Acc. Chem. Res. 48, 380 (2015).

[63] A. S. Disa, M. Fechner, T. F. Nova, B. Liu, M. Först, D. Prabhakaran, P. G. Radaelli, and A. Cavalleri, Polarizing an antiferromagnet by optical engineering of the crystal field, Nat. Phys. 16, 937 (2020). 